\title{
Des vieillards modèles aux jeunes héros dans la
} Florence républicaine

Anziani da imitare e giovani eroi nella Firenze repubblicana del Rinascimento

\section{Cécile Terreaux-Scotto}

\section{OpenEdition}

\section{Journals}

Édition électronique

URL : http://journals.openedition.org/cei/1033

DOI : $10.4000 /$ cei. 1033

ISSN : 2260-779X

\section{Éditeur}

UGA Éditions/Université Grenoble Alpes

\section{Édition imprimée}

Date de publication : 31 octobre 2012

Pagination : 135-168

ISBN : 978-2-84310-234-9

ISSN : 1770-9571

\section{Référence électronique}

Cécile Terreaux-Scotto, "Des vieillards modèles aux jeunes héros dans la Florence républicaine », Cahiers d'études italiennes [En ligne], 15 | 2012, mis en ligne le 30 avril 2014, consulté le 27 mars 2021. URL : http://journals.openedition.org/cei/1033 ; DOI : https://doi.org/10.4000/cei.1033 


\title{
DES VIEILLARDS MODÈLES AUX JEUNES HÉROS DANS LA FLORENCE RÉPUBLICAINE
}

\author{
Cécile Terreaux-Scotto \\ Université Stendhal-Grenoble 3, GERCI
}

"Il faut imiter les grands hommes. C'est le bréviaire de la Renaissance», observe Daniel Ménager en préfaçant l'étude de Patricia Eichel-Lojkine sur Le Siècle des grands hommes ${ }^{\mathrm{I}}$. La ville de Florence n'échappe pas à cette règle. L'objectif de cette étude est de montrer que le choix de ces "grands hommes" à imiter est déterminé par les valeurs qui dominent la société à un moment donné, les valeurs étant, selon une définition empruntée à Jacques Le Goff, «les noyaux valorisés de la pensée et des mentalités ${ }^{2}$ ».

Nous verrons dans un premier temps que pour former leurs enfants à la politique, les marchands du $\mathrm{Xv}^{\mathrm{e}}$ siècle s'appuient sur des figures exemplaires de bons citoyens, dont ils établissent la biographie dans leurs livres de famille. Ces modèles à imiter sont en effet puisés dans l'histoire familiale dans la mesure où les fondateurs du régime des corporations estiment être les seuls à avoir les qualités requises pour gouverner la cité3, qualités qui se transmettent en outre de génération en génération. Les "grands hommes" des familles marchandes sont dès lors leurs ancêtres, qui sont de véritables incarnations de la sagesse. Que les jeunes gens suivent leur exemple et ils sont assurés d'être à leur tour de parfaits citoyens.

Cependant, de I494 à I498, le prédicateur dominicain Jérôme Savonarole exige des Florentins qu'ils renoncent à ce «vieil usage» (vecchia usanza)

I. Daniel Ménager, Préface à Patricia Eichel-Lojkine, Le Siècle des grands hommes. Les recueils de vies d'hommes illustres avec portraits du XVI siècle, Louvain, Peeters, 200I, p. 2.

2. Jacques Le Goff, Héros du Moyen Âge, le Saint et le Roi, Paris, Gallimard, coll. "Quarto», 2004, p. I264. Sur l'héroïsme à la Renaissance, voir Georg Weise, L'ideale eroico del Rinascimento e le sue premesse umanistiche, Naples, Edizioni scientifiche italiane, 1961.

3. Lauro Martines, The Social World of the Florentine Humanists. I390-I460, Londres, Routledge \& Kegan Paul Ltd, 1963, p. 43 et Gene A. Brucker, Florentine Politics and Society (1343-1378), Princeton, Princeton University Press, 1962, p. 87. 
pour adopter une «nouvelle coutume» (nuova consuetudine), condition préalable et nécessaire à la réforme religieuse, morale et politique qu'il appelle de ses voux. Nous analyserons ainsi comment il élabore dans ses sermons un autre ordre des générations, qui substitue à la généalogie charnelle célébrée dans les livres familiaux une généalogie spirituelle, reposant non plus sur les valeurs privilégiées par les marchands mais sur la transmission des vertus chrétiennes ${ }^{4}$. Dans ce contexte, non seulement la sagesse des vieillards et l'autorité qui lui est liée ne vont plus de soi, mais les enfants, qui d'après le prédicateur ont compris quel est le projet de Dieu pour Florence, sont présentés comme des modèles de bons citoyens.

Les jeunes gens pourront dès lors devenir de véritables héros. À un moment où les guerres d'Italie contraignent à évaluer la façon dont ont gouverné les aînés, la jeunesse, à la fois vigoureuse et prévoyante, peut apparaître comme possédant toutes les qualités pour défendre Florence contre les armées étrangères. Au début du Xvi ${ }^{\mathrm{e}}$ siècle, la figure du soldat héroïque qui se sacrifie pour sa patrie estompe ainsi celle du vieux sage.

Efforce-toi aussi de fréquenter un (ou plusieurs) homme de valeur, sage, âgé et sans vice et d'entrer dans son intimité; observe attentivement ses façons de faire, ses mots, ses conseils [...]; apprends à travers lui, forme-toi à travers lui, et suis-le de cette façon et efforce-toi de lui ressembler; aie-le toujours devant toi et à l'esprit, et quand il fait quelque chose, prends exemple sur lui 5 .

Cette recommandation de Giovanni Morelli à ses descendants est emblématique de la façon dont les marchands florentins envisagent l'éducation de leurs enfants au $\mathrm{Xv}^{\mathrm{e}}$ siècle. Conformément à ce que préconisent

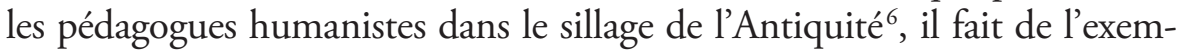
plarité le cœur de l'éducation. Il est en effet connu depuis Aristote, dont les œuvres traduites figurent en bonne place dans les bibliothèques des

4. Sur cette question, Cécile Terreaux-Scotto, " "Vous êtes des enfants de cent ans”. Âge réel et âge métaphorique dans les sermons politiques de Savonarole", Il Pensiero politico, XXXVI, n I, 2003, en particulier p. I4-I7.

5. "Ancora t’ingegna avere usanza e dimestichezza con uno o più valente uomo, savio e antico e sanza vizio, e quello ragguarda ne' modi suoi, nelle parole, ne' consigli [...]; da lui imprendi, da lui appara, e cosi il seguita e t'ingegna di somigliarlo: abbilo sempre innanzi e nella tua mente, e quando fai una cosa ispecchiati in lui." (Giovanni Morelli, I ricordi, dans V. Branca (éd.), Mercanti Scrittori. Ricordi nella Firenze tra Medioevo e Rinascimento, Milan, Rusconi, I986, p. 205.)

6. Eugenio Garin, L'éducation de l'homme moderne. La pédagogie de la Renaissance (I400-I60o), Paris, Fayard, 1968, p. 9I et p. IOO. Dans la civilisation grecque, le jeune noble était formé par les conseils et les exemples d'un aîné qu’il admirait : Jaeger Werner, Paideia. La formation de l'homme grec, Paris, Gallimard, I964, p. Ix; HenriIrénée Marrou, Histoire de l'éducation dans l'Antiquité, Paris, Seuil, I948, p. 33 et p. 62. La société romaine mettait l'accent sur l'utilité des exemples fournis par les parents et les ancêtres. Egle Becchi, "L'Antiquité», dans E. Becchi et D. Julia (dir.), Histoire de l'enfance en Occident, Paris, Seuil, 1998, t. I : De l'Antiquité au $X V I I^{e}$ siècle, p. 52. 
Florentins, que l'imitation est une inclination naturelle de l'homme dès son plus jeune âge ${ }^{7}$.

Une éducation fondée sur l'imitation suppose donc que l'enfant dispose de modèles qui lui éviteront d'être influencé par de mauvais exemples. Mais le modèle préconisé par Morelli n'est pas incarné seulement par un «homme de valeur et sans vice». Il est aussi incarné par un homme «sage et âgé».

Or, l'association de ces deux adjectifs dans la société florentine du $\mathrm{XV}^{\mathrm{e}}$ siècle constitue une tautologie. Car la sagesse provient de la prudence, cette vertu qui consiste, grâce aux enseignements du passé, à comprendre le présent pour mieux prévoir le futur. Dante écrivait à ce propos dans le Convivio que pour être prudent «il faut une bonne mémoire des choses passées, une bonne connaissance des choses présentes, et une bonne anticipation des choses futures ${ }^{8} »$. Parce qu'ils bénéficient d'une expérience acquise au fil des années, la prudence est l'apanage des vieillards. Alberti fait ainsi dire à Benedetto, un des personnages des Libri della famiglia, que les «cheveux [blancs] [1]e rendent prudent et savant en toutes choses"».

Forts d'une parole de vérité qui va de soi et d'une crédibilité qui ne saurait être remise en question, les vieillards bénéficient donc d'une autorité "énonciative» qui leur permet d' "engendrer la croyance et de produire la persuasion", pour adopter les termes qu'emploie Gérard Leclerc dans son Histoire de l'autoritér ${ }^{\circ}$.

Les jeunes générations obéissent dès lors naturellement à leurs aînés auxquels elles sont soumises et qui les protègent de la corruption morale. Les jeunes gens «sont enclins au mal, et s'ils ne sont pas freinés par l'exemple influent des plus vieux, ils tombent facilement dans des vices de plus en plus grands», observe Pier Paolo Vergerio à ce propos ${ }^{\text {II. }}$ "L'âge qui croît

7. Aristote, Poétique, B. Gernez (éd. et trad.), Paris, «Les Belles Lettres», 1997, p. II. Sur la circulation des œuvres d'Aristote à Florence, R. R. Bolgar, The Classical Heritage and its beneficiaries, Cambridge, University Press, 1958, p. 465-468 et Christian Bec, "Recherches sur la culture à Florence», Revue des Études italiennes, nouvelle série, t. XIV, $\mathrm{n}^{\circ} \mathrm{I}$, janvier-mars I968, p. 217.

8. "[...] si richiede buona memoria de le vedute cose, buona conoscenza de le presenti e buona provedenza de le future", Dante Alighieri, Il Convivio, dans Opere minori, C. Vasoli et D. De Robertis (éd.), Milan-Naples, Riccardo Ricciardi, I988, vol. 5, t. I, IV, XXVII, 5.

9. "Questi capelli [canuti] di tutto [mi] fanno prudente e conoscente [...]", Leon Battista Alberti, I Libri della famiglia, R. Romano et A. Tenenti (éd.), Turin, Einaudi, 1969, p. 25.

Io. Gérard Leclerc, Histoire de l'autorité. L'assignation des énoncés culturels et la généalogie de la croyance, Paris, PUF, 1996, p. 7.

II. "[...] sono inclinati al male e, se non vengono raffrenati dall'esempio autorevole dei più vecchi, facilmente cadono in vizi sempre maggiori", E. Garin, Educazione umanistica, Bari, Laterza, 1949, p. 72. 
doit toujours être encouragé et guidé par la prudence des vieux", recommande de son côté Matteo Palmieri ${ }^{\mathrm{I} 2}$.

Inspirée des préceptes éducatifs de l'Antiquitér ${ }^{13}$, cette relation hiérarchique entre vieux et jeunes constitue un des fondements de la vie politique florentine au $\mathrm{Xv}^{\mathrm{e}}$ siècle : comme la prudence est une valeur essentielle de l'art de gouverner, les vieux jouissent aussi d'une autorité institutionnelle, c'est-à-dire d'un "pouvoir légitime» les autorisant à «imposer l'obéissance à ceux qu'il $[s]$ prétend[ent] diriger ${ }^{14} »$.

Par ailleurs, les membres des grandes familles marchandes qui se partagent traditionnellement le pouvoir à Florence évaluent la capacité politique d'un individu d'abord et avant tout à l'aune de l'héritage qu'il a reçu de ses aïeux. De sorte que l'expérience politique est de l'ordre du savoir communicable de génération en génération. C'est pourquoi un jeune homme peut être considéré a priori apte à gouverner dès lors que ses propres ancêtres ont eux-mêmes gouverné : disposant d'un capital de compétences politiques dès la naissance, il possède un "droit naturel à gouverner ${ }^{15}$ ». Significatif est l'exemple de Laurent le Magnifique, appelé à diriger Florence en 1469 alors qu'il a tout juste vingt ans, dans une cité où les charges publiques les plus importantes sont au contraire réservées aux hommes de plus de trente-cinq ans (pour les membres des collèges et du priorat) ou même quarante-cinq ans (pour le gonfalonier de justice).

Dans ces conditions, ce sont leurs propres ancêtres plus qu'un vieillard quelconque que les jeunes gens imiteront. Vergerio remarque ainsi que "les exemples domestiques valent plus que les autres pour nous faire aimer la vertu ${ }^{16}$ » et Palmieri affirme réciproquement que «les mauvaises mours de la maison corrompent beaucoup plus la famille que ne le font celles du dehors ${ }^{17}$ ".

Pour présenter ces modèles familiaux, les marchands florentins disposent d'un outil pédagogique particulièrement efficace : leurs livres de

I2. "La crescente età sempre debbe essere confortata et ferma dalla prudentia de' vechi [...]", Matteo Palmieri, Vita civile, G. Belloni (éd.), Florence, Sansoni, I982, p. 5I.

I3. Jean-Pierre Néraudau, La jeunesse dans la littérature et les institutions de la Rome républicaine, Paris, "Les Belles Lettres", 1979, p. I25 et p. I35.

I4. G. Leclerc, Histoire de l'autorité, ouvr. cité, p. 7.

15. Dale Kent, "The Florentine Reggimento in the Fifteenth Century", Renaissance Quartely, I975, XXVIII, p. 582 .

I6. "[...] gli esempi casalinghi valgono più degli altri a innamorarci della virtù [...]", E. Garin, Educazione umanistica, ouvr. cité, p. Io8.

17. "[...] $i$ costumi tristi di casa molto più corrompono la famiglia che non fanno quegli di fuori [...]", M. Palmieri, Vita civile, ouvr. cité, p. I6o. 
famille, qu'ils se transmettent de pères en fils ${ }^{18}$. Destinés à conserver la mémoire politique d'une lignée, les livres de famille sont essentiellement constitués de galeries de portraits d'ancêtres, pendants littéraires des portraits peints ou des bustes en bois, en cire ou en terre cuite qui sont vénérés dans les maisons ${ }^{19}$.

Mausolées édifiés à la gloire des ancêtres, les livres de famille exposent, à la manière des recueils de vies illustres qui se développent après Pétrarque sur les traces de Plutarque ${ }^{20}$, comment chacun d'entre eux s'est distingué au service de la cité. Les biographies des ancêtres, figures de citoyens exemplaires et emblèmes de la réussite familiale, sont alors pour les jeunes générations de véritables vade-mecum de l'art de gouverner.

Car le livre de famille ne connaît pas de mauvais ancêtre. Un aïeul qui se serait mal comporté est immédiatement puni par le silence : le rédacteur ne parle tout simplement pas de lui! Si bien qu'il tombe dans l'oubli et que les jeunes gens ne risquent pas de suivre un mauvais exemple dont ils ignorent tout. Tel est le sort que Donato Velluti réserve à son cousin Iacopo qui a commis la faute de ne pas avoir de descendance : "Comme il apparaît que le dit lacopo mourut très jeune, sans avoir de femme ni d'enfants, je ne ferai aucune mention de lui ${ }^{21}$. "Giovanni Morelli sanctionne moins sévèrement Calandro, escroc notoire, dont seule la descendance est passée sous un silence aussi désapprobateur que significatif ${ }^{22}$. Mais les rédacteurs de livres de famille font toujours œuvre de censure, célébrant les bons ancêtres et maudissant par l'indifférence les mauvais. Il ne saurait

18. Sur l'écriture des livres de famille, voir entre autres Christian Bec, Les marchands écrivains. Affaires et humanisme à Florence. 1375-I434, Paris, La Haye, Mouton \& Co and École Pratique des Hautes Études, 1967; Vittore Branca, "Mercanti-scrittori fra Trecento e Quattrocento», dans V. Branca, Dizionario critico della letteratura italiana, Turin, U.T.E.T., I986, vol. 2, p. I59-I60 et ID., "Ricordi domestici nel Trecento e nel Quattrocento", dans V. Branca, Dizionario critico della letteratura italiana, ouvr. cité, vol. 3, p. 609-6I2; Angelo Cicchetti et Raul Mordenti, I «libri di famiglia»: problemi di storiografia letteraria e metodologia della ricerca, Rome, Materiali per la didattica e la ricerca, 1983 et ID., "La scrittura dei libri di famiglia", dans A. Asor Rosa, Letteratura italiana, Turin, Einaudi, 1984, vol. III : Le forme del testo, 2 : la prosa, p. III7-II59; Leonida Pandimiglio, "Ricordanza e libro di famiglia. Il manifestarsi di una nuova fonte», Lettere italiane, XXXIX, janvier-mars $1987, \mathrm{n}^{\circ}$ I, p. 3-19.

19. Attilio Schiaperelli, La casa fiorentina e i suoi arredi nei secoli XIV e XV, M. Sframeli et L. Pagnotta (éd.), Florence, Casa Editrice Le Lettere, 1983, vol. I, p. I9I-I93; Lorne Campbell, Portraits de la Renaissance. La peinture des portraits en Europe aux XIV, XV et XVI siècles, s.l., Hazan, I99I, p. 43; Francis William Kent, Household and Lineage in Renaissance Florence. The Family Life of the Capponi, Ginori, and Rucellai, Princeton, New Jersey, Princeton University Press, I977, p. IIO-II2; Charles De la Roncière, «La vie privée des notables toscans au seuil de la Renaissance», dans G. Duby (dir.), Histoire de la vie privée, Paris, Seuil, 1985, t. 2 : De l'Europe féodale à la Renaissance, p. 239.

20. Voir à ce propos P. Eichel-Lojkine, Le Siècle des grands hommes, ouvr. cité., p. 30-34.

2I. "E perché dimostra che 'l detto Iacopo morisse assai giovane, sanza avere moglie o figliuoli, impertanto non farò di lui menzione alcuna [...]", Donato Velluti, La Cronica domestica, scritta fra il 1367 e il I370, con le addizioni di Paolo Velluti, scritte fra il I555 e il I560, I. Del Lungo et G. Volpi (éd.), Florence, Sansoni, I9I4, p. 7. 22. G. Morelli, I Ricordi, ouvr. cité, p. I34. 
en effet être question de perdre du temps à établir la biographie et le portrait d'un individu qui ne peut pas être un modèle à imiter. De fait, comme le souligne Christiane Klapisch-Zuber,

ne passe pas au rang d'ancêtre qui veut : il lui aura fallu se marier, s'être montré prolifique en fils, fidèle aux intérêts et au nom du lignage, ou, si sa propre lignée s'est éteinte par sa faute ou celle de ses proches, avoir acquis des titres particuliers au respect et au souvenir des plus lointains de ses agnats ${ }^{23}$.

Tout ancêtre qui faillirait à son devoir pédagogique étant jeté hors du livre de famille, ceux qui ont échappé à cette sanction constituent nécessairement de bons exemples à suivre. Mieux encore : tous sont de véritables héros ${ }^{24}$.

Puisque les livres de famille sont écrits pour glorifier les ancêtres, ces derniers sont identifiés en tant que héros à travers ce que Philippe Hamon appelle la "prédésignation conventionnelle» : «Ici c'est le genre qui a priori définit le héros ${ }^{25}$." Les ancêtres en sont d'ailleurs aussi les protagonistes stéréotypés, dans la mesure où tous répondent à des critères identiques de sélection.

Puisque les jeunes gens doivent les imiter pour se former au gouvernement de la cité, ils ne peuvent qu'être de sexe masculin. Les femmes sont de fait extrêmement rares dans les livres de famille, et lorsqu'elles y figurent, c'est en raison de leurs qualités viriles. Exceptions qui confirment la règle, Mea Morelli est aussi cultivée qu'un homme et a «l'âme virile ${ }^{26}$ » et Guglielmetta Guicciardini, aux dires de son petit-fils Francesco,

non seulement avait parfaitement toutes les qualités qu'on attend chez une femme, [...] mais elle eut aussi une excellente intelligence et un excellent jugement pour les choses dont les hommes doivent s'occuper ${ }^{27}$.

Les ancêtres mâles qui peuplent les livres de famille appartiennent par ailleurs tous à une époque révolue et sont de toute façon morts la plupart du temps. C'est même généralement au moment de la mort de son père

23. Christiane Klapisch-Zuber, «L'aïeul des généalogies toscanes», Grands-parents, aïeux. Annales de Démographie Historique, I991, p. I05.

24. On trouvera une définition de ce terme dans Philippe Sellier, Le Mythe du héros, Paris-Montréal, Bordas, coll. "Thématique», I970.

25. Philippe Hamon, Pour un statut sémiologique du personnage, dans R. Barthes, W. Kayser, W.C. Booth et Ph. Hamon, Poétique du récit, Paris, Seuil, coll. «Essais», 1977, p. I56.

26. "animo virile», G. Morelli, I Ricordi, ouvr. cité, p. I53. Voir l'analyse de ce portrait dans Ch. Bec, Les marchands écrivains, ouvr. cité, p. 7I-72.

27. " [...] non solo ebbe compiutamente tutte quelle parte si aspettano a una donna, [...], ma ancora ebbe ottimo ingegno e giudicio in quelle cose che si aspettano agli uomini», Francesco Guicciardini, Memorie di famiglia, dans R. Palmarocchi (éd.), Scritti autobiografici e rari, Bari, Laterza \& Figli, I936, vol. 5, p. 29-30. 
que le marchand florentin se met à écrire. Michele Castellani poursuit le travail de rédaction de son père le jour où celui-ci meurt, et il commence son propre livre l'année suivante ${ }^{28}$. Piero Parenti est de son côté autorisé à continuer le livre familial par son père agonisant, comme il l'explique lui-même :

Je rappelle comment le 5 juin [I497], comme Marco fils de Parente fils de Giovanni Parenti était à l'agonie, et me laissait moi Piero, son fils légitime, héritier universel ab intestato, il m’autorisa - je le lui avais demandé -, à continuer à écrire sur tous ses livres, de ma propre main, tout ce qui arriverait; et c'est pourquoi, exécutant sa volonté, je commencerai ici comme on le verra après ${ }^{29}$.

Suivant une ligne de parenté patrilinéaire, le rédacteur qui prend la suite de son père fonde par ce geste son autorité pédagogique sur ses descendants. Il devient à son tour celui qui éduque les générations qui lui succèdent, en tirant profit de l'enseignement qu'il a reçu de ses aïeux.

De sexe masculin, appartenant à une époque révolue, les ancêtres-héros se distinguent enfin par leurs grandes qualités. Les trois quarts des adjectifs se rapportant aux aïeux de la Cronaca domestica par exemple sont positifs et son auteur Donato Velluti proclame, à chaque fois qu'il le peut, leur supériorité morale : alors qu'il condamne la pratique de l'usure de manière générale, il admire en revanche un de ses ancêtres qui en a fait usage ${ }^{30}$. Tout se passe comme si le simple fait d'être identifié en tant qu'ancêtre et d'avoir trouvé place dans le livre familial certifiait la moralité de l'individu et lui conférait une aura de gloire. Giovanni Rucellai célèbre ainsi la noblesse de la maison de son beau-père Palla Strozzi, mais aussi l'abondance de ses richesses, le prestige de sa parenté, la loyauté de ses amis, l'étendue de sa culture et la fécondité de son union conjugale ${ }^{3 \mathrm{I}}$. Quant au beau-père de Francesco Guicciardini, Alamanno Salviati, il bénéficie de qualités significativement semblables ${ }^{32}$.

Tous les portraits mélioratifs des aïeux vont même parfois jusqu’à leur prêter des traits épiques. Ainsi, à cent-trente ans, Ruzza Niccolini était un

28. Giovanni Ciappelli, «I Castellani di Firenze: dall'estremismo oligarchico all'assenza politica (secoli XIVXv)», Archivio Storico Italiano, 547, CXLIX, I99I, disp. I, p. 46.

29. "Ricordo questo di s di giugno come venendo Marco di Parente di Giovanni Parenti in fine di morte, $e$ lasciando me Piero, suo figliuolo legittimo e naturale, suo erede universale ab intestato, mi concesse, domandandonelo io, che seguitassi di scrivere in su tutti e’ suoi libri, di mia mano, secondo che accadessi; e però, esseguendo la sua volontà, farò di qui principio come appresso si mostrerrà", Piero Parenti, Storia fiorentina, A. Mattucci (éd.), Florence, Leo S. Olschki, I994, vol. I, Introduzione, p. Ix.

30. C. De la Roncière, "La vie privée des notables toscans au seuil de la Renaissance», art. cité, p. 267 et V. Branca, Introduzione, dans Mercanti Scrittori, ouvr. cité, p. LII.

3I. Giovanni Rucellai, Il Zibaldone Quaresimale, A. Perosa (éd.), Londres, The Warburg Institute, University of London, I960, p. 54 et p. 63.

32. F. Guicciardini, Ricordanze, dans R. Palmarocchi (éd.), Scritti autobiografici e rari, ouvr. cité, p. 66-68. 
"homme au corps grand et beau ${ }^{33}$ »; de même Bonaccorso Velluti «courageux, fort, vigoureux», «de belle taille, les membres forts, et bien bâti» à cent-vingt ans, «aurait fait se courber n'importe quel jeune plus vigoureux que lui s'il l'avait pris par l'épaule ${ }^{34} »$ ! Ce Bonaccorso a d'ailleurs eu un passé de vaillant guerrier, dont «toutes les chairs étaient recousues, tellement il a eu de blessures dans des batailles et des bagarres ${ }^{35}$ ». Leur âge invraisemblable qui de surcroît va de pair avec une vigueur exceptionnelle fait donc de ces hommes des êtres hors du commun.

C'est pourquoi les jeunes générations sont invitées à accomplir un devoir de répétition destiné à perpétuer les valeurs transmises par leurs aïeux exemplaires. La fin du gouvernement de Pierre de Médicis est a contrario symptomatique de cette exigence qu'ont les Florentins. «Piero, ne persévérant pas dans les habitudes et les avertissements paternels et ne conservant pas les vieux amis de son père, se tourna vers de nouveaux amis", analyse Cerretani ${ }^{36}$. Cette remise en question du modèle paternel irrite rapidement l'oligarchie florentine. Et quand Piero s'empresse de faire bon accueil au roi de France Charles VIII, sans même consulter les membres du gouvernement ${ }^{37}$, sa présence à la tête de la cité devient insupportable. Or, il semble que les Florentins ne pardonnent pas au jeune fils de Laurent le Magnifique de ne pas s'être montré digne de ses prédécesseurs, lui qui avait pourtant hérité de compétences naturelles pour la politique. Luigi Guicciardini raconte à ce propos qu'au cours d'une assemblée réunie le 4 novembre 1494 , de nombreux

citoyens parlèrent en montrant que les façons de Piero leur avaient fortement déplu et qu'elles étaient différentes de celles de ses ancêtres, et que si on n’y remédiait pas la cité serait ruinée par sa faute, et en somme on vit ce jour-là que l'esprit de tous était ennemi de Piero, et que ses façons déplaisaient fortement ${ }^{38}$.

33. "huomo grande del corpo, e bello", Ch. Bec (éd.), Il libro degli affari proprii di casa de Lapo di Giovanni Niccolini de’ Sirigatti, Paris, S.E.V.P.E.N., École Pratique des Hautes Études, 1969, p. 56.

34. "ardito, forte, atante" "di bella statura, e le vembra forti, e bene complesso" "se avesse preso qualunque giovane piu atante in su l'omero, l'avrebbe fatto accoccolare», D. Velluti, La Cronica domestica, ouvr. cité, p. 72.

35. "Tutte le carni sue erano ricucite, tante fedite avea avute in battaglie e zuffe", ibid., p. 72.

36. "Piero, non perserverando ne' chostumi e amonitioni paterne et non perservandosi gl'amici vechchi del padre, si volse a nuovi amici", Bartolomeo Cerretani, Storia fiorentina, Giuliana Berti (éd.), Florence, Leo S. Olschki, 1994, p. I87.

37. Guidubaldo Guidi, Ciò che accadde al tempo della Signoria di novembre dicembre in Firenze l'anno I494, Florence, Arnaud, 1988, p. I3-I7.

38. "[...] ciptadini parlarono mostrando dispiacere loro assai e modi tenuti da Piero et che erano fuori di quelli dei suoi passati, et che se non si rimediava a quelle cose la ciptà rovinerebbe alla [per] sua cagione, et insomma quasi in tutti si vidi quel di l'animo loro proprio inimico a Piero, et dispiaceva loro assai e sua modi", cité par G. Guidi, ibid., p. 26-27. 
Les Florentins reprochent donc à Piero de ne pas avoir su marcher sur les traces de ses ancêtres, son incompétence politique étant à la fois la cause et la conséquence de son incapacité à imiter les modèles que la tradition pédagogique et politique lui imposait pourtant de suivre.

Si le rejet du modèle paternel et ancestral est source de déchéance jusqu'en I494, Savonarole en fait au contraire la condition nécessaire pour la réforme religieuse, morale et politique de Florence.

Le prédicateur fait pourtant de l'imitation la base de toute éducation, comme les marchands florentins. "Toutes nos connaissances commencent par les sens ${ }^{39} "$, explique-t-il. De sorte que tout comme l'aveugle et le sourd de naissance n'ont jamais pu connaître les couleurs et les sons, un individu qui n'est pas exposé à de mauvais exemples ne pourra que suivre les bons ${ }^{40}$. Et étant donné que d'après lui "l'exemple du bien vivre est celui qui convainc tout un chacun ${ }^{4 \mathrm{I}}$ ", chaque Florentin est invité à être un modèle pour les autres :

Dans ce renouveau [...] il faut donner de soi un bon exemple du bien vivre chrétien et que chacun dans tous ses actes montre de la contrition et une vie chrétienne, dans son habillement comme dans toute autre chose [...] il faut que notre vie et notre simplicité soient un exemple pour les autres, et que prêchées aux autres elles les convertissent au bien faire ${ }^{42}$.

Chacun, homme ou femme, vieillard ou enfant, riche ou pauvre, participe ainsi de cette élaboration de modèles de bien vivre, les manifestations extérieures de la conversion individuelle de chaque Florentin conduisant l'ensemble de la cité à la réforme collective ${ }^{43}$. Les parents ont toutefois un rôle particulièrement important à jouer vis-à-vis de leurs enfants ${ }^{44}$, de

39. "[...] ogni nostra cognizione comincia dal senso [...]", Girolamo Savonarola, Triumphus crucis. Testo latino e volgare, M. Ferrara (éd.), Rome, Angelo Belardetti, I96I, I, I, p. 293.

40. G. Savonarola, Prediche sopra Ruth e Michea, V. Romano (éd.), Rome, Angelo Belardetti, I962, V, vol. I, p. I34.

4I. "[...] l'esempio del ben vivere è quello che convince ognuno [...]", G. Savonarola, Prediche sopra Giobbe, R. Ridolfi (éd.), Rome, Angelo Belardetti, I957, XXXIX, vol. 2, p. 267.

42. "In questa reformazione [...] bisogna dare di sé buono esemplo di ben vivere cristianamente e che ciascuno in ogni suo atto mostri compunzione e vita da cristiano, cosi nel vestito come in ogni altra cosa [...] bisogna che la vita nostra e la nostra simplicità sia esemplo agli altri, e predicata agli altri, gli converta al bene operare", G. Savonarola, Prediche sopra Aggeo - Trattato circa el reggimento e governo della città di Firenze, L. Firpo (éd.), Rome, Angelo Belardetti, I965, XI, p. I88-189.

43. Gian Carlo Garfagnini, "Savonarola e la profezia: tra mito e storia», Studi Medievali, serie terza, XXIX, I988, fasc. I, p. I80.

44. G. Savonarola, Prediche sopra Giobbe, ouvr. cité, XXXIX, vol. 2, p. 267. Voir aussi ibid., XXVIII, vol. 2, p. 76 ; XI, vol. I, p. 192 et G. Savonarola, Prediche sopra Aggeo, ouvr. cité, IX, p. I50 et XIV, p. 23 I. 
même que les vieux vis-à-vis des jeunes ${ }^{45}$, les prêtres vis-à-vis des laïcs ${ }^{46}$ ou encore les dirigeants vis-à-vis des citoyens ${ }^{47}$. Le processus d'imitation repose donc sur l'identification d'un individu à un autre au sein d'un rapport hiérarchique binaire. Par un effet de miroir, chaque Florentin peut projeter sur un père, un aîné, un religieux, un magistrat, tous exemplaires, ce qu'il pourrait et devrait être. Cela est particulièrement vrai des enfants, qui "apprennent ce qu'ils entendent et ce qu'ils voient ${ }^{48}$ ", souligne Savonarole en paraphrasant Aristote ${ }^{49}$.

Au huis-clos familial des livres marchands doit donc se substituer un élan collectif de tous les citoyens florentins vers la réforme. Significativement, Savonarole souhaite que le nom des membres de la seigneurie qui ont mis en place les nouvelles institutions de I494 «soient inscrits dans les chroniques [des Florentins] pour servir d'exemple aux autres qui viendront, afin qu'ils se comportent bien ${ }^{50}$ ". Si l'éducation à la politique repose toujours sur l'exemplarité et si les modèles à imiter sont toujours ceux de bons citoyens, en revanche les ancêtres ne constituent plus à eux seuls les réservoirs d'exemples dans lesquels les jeunes gens sont invités à puiser.

De fait, le prédicateur répète inlassablement que les Florentins éduquent mal leurs héritiers parce qu'ils n'ont pas encore renoncé à leurs traditions, au "vieil usage» qui fait obstacle à la réforme. La transmission de l'expérience politique de génération en génération ne va donc plus de soi. Elle est même radicalement remise en cause par un prédicateur qui considère que la sagesse ne trouve pas son origine dans la prudence, cette combinaison de la mémoire, de l'intelligence et de la prévision, mais dans le «bien vivre».

Maîtriser le passé, le présent et le futur est en effet inutile pour une réforme dont le temps n'est pas celui de l'homme, mais celui de Dieu, comme il apparaît dans les Actes des Apôtres (I, 7). «Seule l'expérience du bien vivre fera qu'on t'estimera prudent, et non tes cheveux blancs», lance ainsi Savonarole aux vieillards florentins ${ }^{\text {sI }}$. Don de Dieu, la sagesse ne peut

45. G. Savonarola, Prediche sopra Aggeo, ouvr. cité, XIV, p. 242 et G. Savonarola, Prediche sopra Giobbe, ouvr. cité, IX, vol. I, p. I66.

46. G. Savonarola, Prediche sopra Giobbe, ouvr. cité, XXXIX, vol. 2, p. 267.

47. G. Savonarola, Prediche sopra Aggeo, ouvr. cité, V, p. 83.

48. "[...] imparano quello che odano e vedano [...]", G. Savonarola, Prediche sopra Ruth, ouvr. cité, VIII, vol. I, p. 228.

49. Aristote, Poétique, ouvr. cité, p. II-I3.

50. "[...] che siano scritti in su le vostre croniche per dare esempio alli altri, che verranno, di portarsi bene [...] ", G. Savonarola, Prediche sopra Aggeo, ouvr. cité, XXIII, p. 426.

5I. "[...] l'esperienzia della buona vita è quella sola che ti farà riputar prudente, non li capelli canuti [...]", G. Savonarola, Prediche sopra Amos e Zaccaria, P. Ghiglieri (éd.), Rome, Angelo Belardetti, I97I, XXV, vol. 2, p. 198. 
qu'être atemporelle et n'est donc pas un privilège accordé par le temps : «Si la sagesse était dans les cheveux blancs, moi aussi je me ferais faire des cheveux blancs ", renchérit un frère Jérôme ironique ${ }^{52}$.

Dans ces conditions, le Dominicain invite les Florentins à «ne plus être semblables à leurs pères passés ${ }^{33}$ » qui leur ont fait perdre leur forme naturelle en leur transmettant l'amour de l'ambition, du superflu et du luxe, ces «idoles» qui les ont éloignés du Christ ${ }^{54}$.

Par ailleurs la sagesse humaine, qui provient de la raison naturelle, doit s'accompagner de la lumière surnaturelle, c'est-à-dire de la grâce donnée par la foiss. Car le renouvellement de la grâce permettra aux Florentins de retrouver leur forme naturelle, qu'ils ont perdue à cause de la seconde forme que l'histoire leur a donnée à travers la coutume ${ }^{56}$.

Or, cette forme naturelle désigne l'état privilégié dans lequel vivaient les patriarches bibliques et les premiers chrétiens, tandis que la seconde forme représente l'éducation que les Florentins ont reçue. Et puisque d'après le prédicateur «la vraie sagesse est le Crucifix : là dans ce livre on apprend toute la véritable sagesse ${ }^{57}$ ", les modèles à imiter seront "ceux du calendrier, et certainement pas ceux que tu appelles nos vieux et nos ancêtres», prévient-il ${ }^{58}$. L'exemple des premiers chrétiens et, plus largement, des saints doit donc remplacer le savoir habituellement transmis par les ancêtres.

Il n'est certes pas nouveau qu'un religieux appelle ses ouailles à imiter les premiers chrétiens. Dans sa Regola del governo di cura familiare, Dominici recommandait ainsi à plusieurs reprises aux parents de s'inspirer de la façon dont les "anciens» (antichi) éduquaient leurs enfants 59 . Il les incitait également à orner leur maison de tableaux pieux afin que les enfants puissent devenir de bons croyants en s'identifiant aux saints et

52. "Se la sapienzia stesse nelli capelli bianchi, io mi farei fare ancora io e’ capelli bianchi», G. Savonarola, Prediche sopra Ruth, ouvr. cité, XI, vol. I, p. 332.

53. "[...] non vogliate esser simili a’ padri vostri passati [...]», G. Savonarola, Prediche sopra Amos, ouvr. cité, XXXIII, vol. 2, p. 425 .

54. Ibid., VI, vol. I, p. I78; voir aussi G. Savonarola, Prediche sopra Ezechiele, R. Ridolfi (éd.), Rome, Angelo Belardetti, I955, XXIV, vol. I, p. 317.

55. G. Savonarola, Prediche sopra Aggeo, ouvr. cité, XXII, p. 387. Sur la condamnation de la sagesse humaine, voir Armando F. Verde, o. p., Lo studio fiorentino. I473-I503. Ricerche e documenti, Florence, Leo S. Olschki, I985, IV, 3, p. II73-II76.

56. Sur cette question, John G. A. Pocock, Le moment machiavélien, Paris, PUF, 1997, p. I19-I25.

57. "[...] la vera sapienzia è el Crucifisso: quivi in quello libro si impara ogni vera sapienzia", G. Savonarola, Prediche sopra Aggeo, ouvr. cité, V, p. 84.

58. "[...] quelli del calendario, non già quelli che tu chiami e’ nostri vecchi e li nostri antichi», G. Savonarola, Prediche sopra Giobbe, ouvr. cité, XLI, vol. 2, p. 315.

59. Giovanni Dominici, Regola del governo di cura familiare, D. Salvi (éd.), Florence, Angiolo Garinei, I860, p. I34, I36, I39 et I44. 
aux saintes ${ }^{60}$. Mais chez Savonarole, cette recommandation s'inscrit non seulement dans un projet politique mais aussi dans une remise en cause de l'ordre traditionnel des générations.

Car ces modèles de vertu que sont les premiers chrétiens, les saints et les martyrs renvoient à un passé universel et atemporel opposé à la succession familiale et rythmée des générations. Emblématique à cet égard est l'allégorie du sermon XXII sur Job où Savonarole dispose sur le trône de Salomon plusieurs saints, qui sont autant d'incarnations exemplaires de la vie ecclésiastique ou familiale. Sur la première marche, les parents de saint Jean-Baptiste et les parents de la Vierge, qui d'après l'Évangile de saint Luc ont toujours respecté les commandements de Dieu $(\mathrm{I}, 6)$, donnent l'exemple d'une vie familiale digne d'être imitée. Ces deux couples prennent ainsi implicitement la place des figures parentales qui étaient jusque-là proposées aux jeunes générations dans les livres de famille. Une marche plus haut, c'est la vie dans l'Église qui est représentée d'un côté par sainte Catherine, vierge et martyre qui a prêché pour l'amour de Dieu contre les philosophes païens du Iv siècle, et de l'autre par sainte Catherine de Sienne qui, au moment du schisme de l'Église au XIV siècle, a convaincu Grégoire XI de replacer le Saint-Siège à Rome.

Les occupants de la troisième marche sont particulièrement adaptés à l'auditoire florentin de Savonarole. Saint Antonin, prieur de San Marco à partir de I439, a cherché à remettre de l'ordre dans les mours corrompues du clergé. Sa biographie était connue grâce à Vespasiano da Bisticci qui en avait dressé dans son recueil de vies illustres un portrait vantant la pureté de ses mœurs ${ }^{6}$. Ce choix souligne la volonté de frère Jérôme de remplacer les galeries d'ancêtres des livres de famille par des portraits de Florentins pouvant participer à l'élan collectif en faveur de la réforme : comme les magistrats qui ont participé à la réforme de I494, saint Antonin a œuvré pour le bien commun de la cité. À côté d'Antonin, san Zanobi, fondateur spirituel de l'évêché florentin au $\mathrm{v}^{\mathrm{e}}$ siècle, est le promoteur de la christianisation définitive de la cité ${ }^{62}$. Il incarne ainsi un modèle d'engagement en faveur du Christ, qui est renforcé par saint Étienne et saint Sébastien. Ces

6o. Ibid., p. I3I. Sur cette fonction pédagogique de l'image, Pierre Riché, Danièle Alexandre-Bidon, L'enfance au Moyen Âge, Paris, Seuil, Bibliothèque nationale de France, 1994, p. II3-II4.

6I. Vespasiano da Bisticci, Vite di uomini illustri del secolo XV, P. d'Ancona et E. Aeschlimann (éd.), Milan, Ulrico Hoepli, I95I, p. I29 et I34.

62. Anna Benvenuti Papi, «S. Zanobi: memoria episcopale tradizioni civiche e dignità familiari», dans I ceti dirigenti nella Toscana del Quattrocento. Comitato di studi sulla storia dei ceti dirigenti in Toscana (Atti del V e VI Convegno, Florence, IO-II décembre I982; 2-3 décembre 1983), Florence, Francesco Papafava, I987, p. 79-II5. 
deux martyrs, placés sur la quatrième marche, suggèrent que les Florentins doivent être eux aussi prêts à sacrifier leur vie pour réformer l'Église ${ }^{63}$.

Encore plus haut, saint Jean l'Évangéliste et saint Marc montrent aux Florentins comment répandre la Bonne Nouvelle. Enfin, le prophète David et saint Jean Baptiste évoquent sur la dernière marche du trône le renouveau et l'alliance avec Dieu auquel tous les Florentins sont appelés : roi idéal aux yeux du peuple d'Israël, David a transféré l'Arche de l'alliance à Jérusalem et Jean Baptiste a montré le chemin de la pénitence au peuple du Seigneur ${ }^{64}$.

En gravissant progressivement ce trône, dont la forme évoque significativement un parcours de formation, les Florentins, forts de l'enseignement des "pères anciens" (padri antichi) qu'ils découvriront petit à petit, parviendront à obtenir l'intercession de la Vierge auprès du Seigneur ${ }^{65}$. Car tous méritent d'être imités, tandis que les vieux, qui ont préféré vénérer des «idoles», deviennent des contre-exemples.

C'est pourquoi Savonarole élabore, au fil des sermons, les portraits exemplaires des premiers chrétiens, dont l'existence simple, charitable et humble, peut et doit servir de modèle aux Florentins, du berceau jusqu'au tombeau.

Pratiquant l'abstinence après la naissance de leurs enfants, les patriarches bibliques permettaient judicieusement aux jeunes accouchées de nourrir elles-mêmes les nouveaux-nés, ce qui est bien préférable, du point de vue de Savonarole, à l'allaitement mercenaire ${ }^{66}$. Lorsque leurs enfants avaient grandi, les premiers chrétiens savaient aussi les préserver des mauvais exemples, alors que les Florentins n'ont aucune pudeur devant leurs enfants qu'ils savent pourtant influençables :

aux temps anciens on vivait partout simplement, on ne parlait pas de choses malhonnêtes et on n'y pensait pas. Aujourd'hui le monde a versé dans une telle folie que les femmes parlent de tout ouvertement avec leurs maris, en présence de leurs fils et de

63. Savonarole invite au martyre par exemple dans Prediche sopra Ruth, ouvr. cité, II, vol. I, p. 55.

64. 2 Samuel 6 et Évangile selon saint Luc, I, 76-77.

65. G. Savonarola, Prediche sopra Giobbe, ouvr. cité, XXII, vol. I, p. 422. Preuve de son importance, cette allégorie est reprise dans le Compendio di rivelazioni. G. Savonarola, Compendio di rivelazioni, testo volgare e latino - Dialogus de veritate prophetica, A. Crucitti (éd.), Rome, Angelo Belardetti, 1974, p. 86-92.

66. G. Savonarola, Prediche sopra Amos, ouvr. cité, XLII, vol. 3, p. 204. Sur la pratique de la mise en nourrice à Florence, Ch. Klapisch-Zuber, "Parents de sang, parents de lait", dans La maison et le nom. Stratégies et rituels dans l'Italie de la Renaissance, Paris, École des Hautes Études en Sciences Sociales, 1990, p. 263-289 et David Herlihy et Ch. Klapisch-Zuber, Les Toscans et leurs familles, Paris, Presses de la Fondation Nationale des Sciences Politiques, Éditions de l'École des Hautes Études en Sciences Sociales, 1978, p. 555-558. 
leurs filles : ton enfant est là et il entend ce dont tu parles, et il commence à laisser la simplicité de côté et à devenir empli de malice ${ }^{67}$.

La simplicité exemplaire des premiers chrétiens apparaît également dans leur renoncement au superflu et dans leur sobriété vestimentaire ${ }^{68}$. Enfin, alors que les Florentins érigent la pratique du commerce en vertu civique qui confère un statut social élevé aux familles qui l'exercent ${ }^{69}$, Savonarole les appelle à renoncer à en montrer les fruits, parce que l'étalage des richesses est contraire à l'esprit de pauvreté du Christ ${ }^{70}$. Savonarole cherche ainsi à briser une tradition pourtant bien ancrée à Florence de la transmission de l'art du commerce de père en fils, qu'il veut remplacer par «l'expérience du bien vivre»:

Viens ici : l'art du bien vivre est l'art le plus excellent qui soit, parce qu'il te fait lier d'amitié avec le Christ. Fais en sorte de l'enseigner à ton fils; cet art est le plus utile qui soit, parce qu'il te conduit à la vie éternelle. [...] Tu as très bien étudié l'art du change, qui est si subtil que les docteurs le comprennent à peine. Mes enfants, n'apprenez pas cet art de vos pères ${ }^{71}$.

Cette dernière injonction adressée à la jeunesse ne peut pas mieux résumer la remise en cause radicale par frère Jérôme des modèles éducatifs traditionnels à Florence. La leçon est d'ailleurs parfois bien comprise, comme le montre l'exemple de Pandolfo Rucellai et Francesco Gondi qui, issus de grandes familles marchandes, abandonnent leur métier de banquiers pour rejoindre les Dominicains de San $\mathrm{Marco}^{72}$.

67. "Al tempo antiquo si viveva per tutto semplicemente, che non si parlava né pensava a una disonestà. [...] $\dot{E}$ oggi venuto el mondo in tanta pazzia che le donne con li mariti, in presenzia de' figliuoli e figliuole, parlano ogni cosa apertamente: sta là el tuo fanciullo e intende quello che tu parli, e comincia a lasciare la simplicità e diventare pieno di malizia", G. Savonarola, Prediche sopra Ruth, ouvr. cité, V, p. I35. Voir aussi Prediche sopra Amos, ouvr. cité, XIII, p. 35I.

68. G. Savonarola, Prediche sopra Giobbe, ouvr. cité, XX, vol. I, p. 382. Voir aussi ID., De simplicitate christiane vite, P. G. Ricci (éd.), Rome, Angelo Belardetti, I959, p. 220-22I.

69. L. Martines, The Social World of the Florentine Humanists, ouvr. cité, p. $30-34$ et D. Kent, «The Florentine Reggimento in the Fifteenth Century", art. cité, p. 58I et p. 596-600.

70. G. Savonarola, Prediche sopra Aggeo, ouvr. cité, IX, p. I52 et VII, p. II8, ainsi que ID., Prediche sopra Ruth, ouvr. cité, IV, vol. I, p. I25 et V, vol. I, p. I34.

7I. "Vien qua: l'arte del ben vivere è la più eccellente arte che sia, perché la ti fa venire in amicizia con Cristo. Questa fa che tu la insegni al tuo figliuolo; questa arte è la più utile che sia, perché la ti conduce in vita eterna. [...] Tu hai molto bene studiato ne l'arte de' cambi, che è cosi sottile ch'e' dottori non la intendano apena. Figliuoli miei, non la imparate quella arte da' padri vostri», G. Savonarola, Prediche sopra Amos, ouvr. cité, VI, vol. I, p. 176.

72. Vanna Arrighi, "Gli adepti del Savonarola. Linee per una ricerca negli archivi familiari», dans G. C. Garfagnini (dir.), Studi savonaroliani. Verso il V centenario, Florence, Sismel «Edizioni del Galluzzo», I996, p. 42. 
Au lieu d'accumuler des richesses, continue frère Jérôme, les Florentins feraient mieux de les transformer en aumônes pour les pauvres ${ }^{73}$. Voilà qui les rendrait dignes des premiers chrétiens qui proscrivaient l'usure, conformément aux préceptes bibliques ${ }^{74}$. Sur ce point, Savonarole fait une fois de plus des «saints du calendrier» les modèles à imiter tandis que les Florentins apparaissent comme des exemples à rejeter :

Aujourd'hui [...] chacun cherche son profit et n'écoute pas sa conscience comme il le faudrait; ce n'était pas ainsi au temps de nos docteurs anciens, qui ne savaient alors probablement pas ce qu'étaient les changes et ces autres combines, qu'on a trouvés après 75 .

Exemplaires pour leur simplicité et leur charité, les premiers chrétiens le sont aussi pour leur humilité. Or, Savonarole présente cette vertu comme nécessaire au nouveau gouvernement créé en I494. Le citoyen humble, qui accorde plus d'importance au bien commun qu'à l'intérêt particulier, ne cherche en effet pas à s'élever au-dessus des autres, contrairement à ce qu'ont fait les Médicis par exemple, qui ont favorisé leurs amis par une politique de clientélisme et instauré le gouvernement d'un seul homme, ce qui ne pouvait qu'aboutir à la tyrannie dans le contexte florentin, la tyrannie favorisant la pratique de tous les péchés ${ }^{76}$. Les premiers chrétiens constituent donc un modèle non seulement moral, mais également politique.

Pour finir, le prédicateur invite les Florentins à imiter les saints qui reposent dans des tombeaux sobres à l'image de leur vie exemplaire, plutôt que de vouloir parader, même une fois morts, dans des tombes somptueuses qui reflètent une vie de cérémonies extérieures sans véritable élan d'amour vers Dieu ${ }^{77}$. Or à Florence, l'édification de chapelles sépulcrales richement ornées n'est pas uniquement un signe d'honneur et de richesse qui matérialise la contribution du donateur à la construction de l'église à

73. Les invitations en ce sens sont multiples. Voir G. Savonarola, Prediche sopra Aggeo, ouvr. cité, I, p. Io-II et p. 20 ; II, p. 42; III, p. 58; V, p. 83; VII, p. II8; VIII, p. I4I ; IX, p. I5I-I53; X, p. I69; XI, p. I76; XIII, p. 220.

74. Sur la pratique de l'usure par les marchands florentins, L. Martines, The Social World of the Florentine Humanists, ouvr. cité, p. 27 et 76; Raymond De Roover, Il banco Medici dalle origini al declino (I397-I494), Florence, «La Nuova Italia» Editrice, ristampa anastatica 1988, p. I8; Iris Origo, Le marchand de Prato. Francesco di Marco Datini, Paris, Albin Michel, 1959, p. I47-I53 et Francesco Cognasso, L'Italia nel Rinascimento, Turin, UTET, 1965, vol. I, p. 709-79I. Sur la position de l'Église à ce sujet, R. De Roover, ouvr. cité, p. I2-I5.

75. "Oggi, [...] ognuno va cercando quello che gli torna piu utile alla robba e non guarda alla conscienzia, come bisognerebbe; non era cosi al tempo di quelli nostri dottori antiqui, che non si sapeva quasi allora che cosa fussero cambi e quest'altre involture, che si sono trovate di poi», G. Savonarola, Prediche sopra Aggeo, ouvr. cité, X, p. 162.

76. Ibid., V, p. 8I-84; IX, p. I45; X, p. 166 et I68 et XII, p. 206. Voir aussi G. Savonarola, Trattato circa il reggimento, ouvr. cité, I, 3 et II, 2.

77. G. Savonarola, Prediche sopra Aggeo, ouvr. cité, II, p. 35-39. 
laquelle sa famille est traditionnellement rattachée ${ }^{78}$. Il s'agit aussi d'accueillir la dépouille de toutes les générations d'une famille et de vénérer les ancêtres à travers l'entretien de l'édifice ${ }^{79}$. La tombe symbolise par conséquent l'unité du lignage autour d'un ancêtre commun ${ }^{80}$. De sorte que les remarques de Savonarole n'invitent pas seulement les Florentins à montrer une humilité toute chrétienne devant la mort. Dans cette invitation à imiter les pratiques mortuaires des saints, il y a aussi un appel à renoncer au culte d'ancêtres qui chérissent l'ambition, le superflu et le luxe, qui sont des «idoles» les détournant des vertus chrétiennes.

Ainsi, la comparaison systématique entre le passé des premiers chrétiens et le présent des Florentins aboutit toujours à la supériorité du premier. C'est pourquoi, dans une société où l'imitation des ancêtres non seulement constitue le cœur de toute éducation, mais détermine aussi en grande partie la légitimité des individus à s'occuper de politique, Savonarole recommande d'abandonner ces modèles familiaux pour les remplacer par «les choses et les vies des saints ${ }^{81} »$. Le récit des exploits et du cursus honorum des aïeux est du côté du "vieil usage», les Florentins doivent y renoncer au profit de l'enseignement des premiers chrétiens qui conduit au renouveau ${ }^{82}$. Une généalogie spirituelle se substitue dès lors à la généalogie charnelle, comme le suggère le chant que Savonarole met dans la bouche des Pères de l'Église qui s'adressent aux Florentins en établissant avec eux un lien significatif de paternité : "nos patres et vos filii ${ }^{83}$ ».

Les efforts répétés de Savonarole pour qu’au «vieil usage» incarné par les ancêtres charnels se substitue la «nouvelle coutume» pratiquée par les pères spirituels sont récompensés quelques mois après le début de sa prédication. D'après le témoignage de Iacopo Nardi, " en ce temps-là on vivait dans notre cité bien plus chrétiennement que dans les temps passés ${ }^{84}$ ». Le

78. L. Martines, The Social World of the Florentine Humanists, ouvr. cite, p. 23 et Richard A. Goldthwaite, La costruzione della Firenze rinascimentale. Una storia economica e sociale, Bologne, Il Mulino, I984, p. I46. Sur le patronage des Florentins sur les églises, Roberto Bizzocchi, "Chiesa e aristocrazia nella Firenze del Quattrocento", Archivio Storico Italiano, 520, CXLII, 1984, disp. II, p. 193-200.

79. F. Kent, Household and Lineage in Renaissance Florence, ouvr. cité..., p. 99-Ioo et p. II3; R. Goldthwaite, La costruzione della Firenze rinascimentale, ouvr. cité, p. I47-I48.

8o. L. Martines, The Social World of the Florentine Humanists, ouvr. cité, p. 57; F. Kent, Household and Lineage in Renaissance Florence, ouvr. cité, p. 272; L. Pandimiglio, "Giovanni di Pagolo Morelli e la continuità familiare", Studi Medievali, serie III, XXII, juillet I98I, p. I60-I62; C. De la Roncière, "La vie privée des notables toscans au seuil de la Renaissance", art. cité, p. 238.

8I. "le cose e le vite de’ santi», G. Savonarola, Prediche sopra Aggeo, ouvr. cité, IV, p. 69.

82. Ibid., IV, p. 69 et G. Savonarola, Prediche sopra Giobbe, ouvr. cité, XXVIII, vol. 2, p. $7 \mathrm{I}$.

83. G. Savonarola, Prediche sopra Amos, ouvr. cité, XXXIV, vol. 3, p. I6-I7.

84. "[...] si viveva in quel tempo nella nostra città molto più cristianamente a comparazione de' tempi passati", Iacopo Nardi, Istorie della città di Firenze, A. Gelli (éd.), Florence, Successori Le Monnier, I888, vol. I, XVI, p. 79 . 
prédicateur regrette cependant que certains Florentins refusent de cesser d'imiter leurs ancêtres. Il le constate en ces termes le 4 avril I495: "Il y a certaines personnes ici, dans ta cité, qui ne veulent en aucune façon renoncer à l'autorité de leurs prédécesseurs et de leurs vieux pères ${ }^{85}$." Mais d'un autre côté, moins d'un an plus tard, il proclame que les jeunes générations ont compris, contrairement à leurs aînés, qu'il fallait désormais suivre l'exemple des premiers chrétiens qu'il met en avant dans ses sermons et non des ancêtres dont les valeurs sont exaltées dans les livres de famille. C'est pourquoi il considère en octobre 1495 que les enfants et les adolescents florentins sont «réformés».

Au lendemain de la première procession des fanciulli dans les rues de la ville, le I7 février I496, Savonarole estime ainsi qu'ils font de Florence une nouvelle Jérusalem : débarrassés de toutes leurs anciennes et mauvaises habitudes, ils ne pensent désormais qu’à louer Dieu ${ }^{86}$. Mais ce n'est pas tout. Il félicite aussi les fanciulli pour avoir compris, contrairement aux générations qui les précèdent, quel était le projet politique de Dieu pour Florence :

Ils sont ceux qui jouiront des grâces de Florence et qui la gouverneront bien, parce qu'ils n'auront pas pris le mauvais pli, comme l'ont pris leurs pères, qui ne peuvent pas se détacher du régime tyrannique et qui ne savent pas reconnaître combien est grande la grâce de la liberté ${ }^{87}$.

En proclamant le Christ roi de la cité, poursuit le prédicateur, les fanciulli "comme des vieux sages [...] ont appris à qui devait leur apprendre ${ }^{88}$ ". Autrement dit, les rôles s'inversent entre les générations d'une part, et entre maîtres et disciples d'autre part. Alors que les aînés sont traditionnellement détenteurs d'une autorité naturelle qui fait d'eux des modèles pour leurs cadets, ce sont désormais au contraire les plus jeunes qui montrent la voie à suivre.

Puisque les fanciulli n'ont pas eu le temps de vieillir sous un mauvais gouvernement et qu'ils ont de toute façon spontanément renoncé au «vieil

85. "[...] sono alcune persone qua, nella tua città, che a nessun modo vogliano lasciare l'autorità de' predecessori loro e delli loro padri antichi», G. Savonarola, Prediche sopra Giobbe, ouvr. cité, XXXIII, vol. 2, p. I43.

86. G. Savonarola, Prediche sopra Amos, ouvr. cité, I, vol. I, p. 37.

87. "Questi saranno quelli che goderanno le felicità di Firenze e la governeranno bene, perché non aranno presa la piega del ciambellotto, come hanno presa i padri loro, che non si possono spiccare dal reggimento tirannico né sanno conoscere quanta è grande questa grazia della libertà", ibid., I, vol. I, p. 38. Sur la dimension politique de la réforme des enfants, C. Terreaux-Scotto, "La place des enfants dans la réforme savonarolienne de la cité ", dans A. Fontes, J.-L. Fournel et M. Plaisance (dir.), Savonarole : enjeux, débats, questions (Actes du colloque international, Paris, 25-26-27 janvier 1996), Paris, Université de la Sorbonne Nouvelle, I998, p. 8I-I03.

88. "come vecchi di senno [...] hanno insegnato a chi doveva insegnare a loro", G. Savonarola, Prediche sopra Amos, ouvr. cité, I, vol. I, p. 39. 
usage» au profit d'une "nouvelle coutume», ils sont en effet supérieurs aux adultes, comme Jésus qui en avait remontré aux docteurs du Temple et même à ses propres parents (Luc, 2, 46-50).

Les enfants louent d'ailleurs le Seigneur sans artifice. Savonarole écrit à ce propos, à la fin de l'année I495, que

notre expérience nous a montré que les actes et les paroles des enfants charment tout le monde parce qu'ils ne recèlent rien d'artificiel ni de faux, mais qu'ils jaillissent naturellement de leur âme sans tache ${ }^{89}$.

Quelques semaines plus tard, à partir du carnaval de février I496, le prédicateur s'inspire d'un verset de Matthieu pour observer à plusieurs reprises que «les sages n'ont pas su le louer, les philosophes n'ont pas su le louer, mais les enfants t'ont loué, ô notre Seigneur. Ex ore infantium et lactentium perfecisti laudem ${ }^{\circ} "$. Significativement, le chroniqueur Luca Landucci, dont les propres enfants font partie du cortège, emploie la même formulation que Savonarole pour décrire l'impression produite par les fanciulli sur le reste de la population :

Et il nous semblait voir les foules de Jérusalem qui venaient à la rencontre du Christ et le suivaient le dimanche des Rameaux, en disant : "Béni soit celui qui vient au nom du Seigneur. Et on peut dire la parole des Écritures : Infanzium e lattenzium perfecisti lalde ${ }^{9 \mathrm{I}} »$.

Si bien que les fanciulli florentins, d'un âge plus ou moins compris entre six et vingt ans, savent louer Dieu avec la même simplicité que les premiers chrétiens, à propos desquels Savonarole observe que

la simplicité et la bonté de nos pères anciens étaient grandes, car, bien qu'ils fussent savants et sages, tamen il régnait en eux une telle simplicité qu'ils croyaient immédiatement et fortement aux choses de Dieu ${ }^{92}$.

89. "E noi ancora per propria esperienzia veggiamo che gli atti e le parole de' fanciulli delettono ciascuno, perché non hanno in loro alcuna arte o duplicità, ma procedono naturalmente dalla loro forma purissima", G. Savonarola, De simplicitate christiance vite, ouvr. cité, III, p. 192. D'abord rédigé en latin, ce texte a été traduit en vulgaire par Savonarole lui-même.

90. "Non l'hanno saputo laudare li savi, non l'hanno saputo laudare li filosofi, ma li fanciulli t'hanno laudato, o Signore nostro. Ex ore infantium et lactentium perfecisti laudem", G. Savonarola, Prediche sopra Amos, ouvr. cité, XL, vol. 3, p. I55. Voir aussi ibid., VII, vol. I, p. 208; XLI, vol. 3, p. I84; G. Savonarola, Prediche sopra Ezechiele, ouvr. cité, XXVI, vol. I, p. 345-346. Voir Matthieu, 2I, I6 ( De la bouche des enfants et des nourrissons tu t'es formé une louange", 2I, I6).

91. "E ci pareva di vedere quelle turbe di Gierusalem ch'andavano inanzi e dietro a Cristo la domenica d'ulivo, dicendo: Benedetto sia tu che vieni nel nome del Signore. E ben si può dire le parole della Scrittura: Infanzium e lattenzium perfecisti lalde», Luca Landucci, Diario fiorentino dal I450 al ISI6 continuato da un anonimo fino al I542, I. Del Badia (dir.), Florence, Sansoni, ristampa anastatica I985, p. I25.

92. "Era grande la simplicità e la bontà dè nostri padri antichi, che benchè fussino dotti e sapienti, tamen regnava in loro tanta simplicità che erano molto creduli subito alle cose di Dio", G. Savonarola, Prediche sopra Aggeo, ouvr. cité, VII, p. I07. Voir aussi ibid., VII, p. I08-I09 et XVIII, p. 303-304. 
C'est pourquoi les fanciulli sont désormais présentés comme des modèles à imiter, à l'instar des premiers chrétiens. La façon dont frère Jérôme s'adresse aux Florentins à ce propos ne peut pas être plus explicite : «Ô vous les vieux, apprenez de ces enfants à louer le Seigneur ${ }^{93}$."

En novembre I494, Savonarole avait rappelé que «le Seigneur protège les petits : les petits et les tout-petits sont ceux qui s'humilient et se rabaissent et se conduisent simplement ${ }^{94}{ }^{\prime}$. Il faisait alors allusion à l'attitude spirituelle des pauvres de l'Ancien Testament, qui n'aiment que Dieu et ne comptent que sur lui, reconnaissant humblement leur ignorance et attendant tout de Dieu, comme un tout-petit qui a besoin d'apprendre et qui attend tout de ses parents ${ }^{95}$. S'appuyant sur les Évangiles, il avait en même temps recommandé aux Florentins d'adopter une attitude semblable, d'abandon et de confiance :

$\mathrm{Si}$ vous ne devenez pas tout petits, vous n'entrerez pas dans le Royaume des Cieux; c'est pourquoi donnez-vous, dis-je, à la simplicité et à l'humilité, car le Seigneur sera avec vous ${ }^{96}$.

À partir du carnaval I496, ce sont les enfants et les jeunes adolescents florentins qui, renonçant aux vices pour acclamer le Christ Roi de la cité, ont adopté cette attitude des simples, comme s'en réjouit à plusieurs reprises le Dominicain ${ }^{97}$. L'enfance entendue comme classe d'âge coïncide dès lors avec l'enfance spirituelle qui ouvre le Royaume des Cieux. Par conséquent, lorsque quelques semaines avant sa mort Savonarole affirme que "celui qui veut entrer dans la vie éternelle doit devenir enfant ${ }^{98}$ ", il veut dire qu'il ne s'agit plus seulement pour les Florentins de se faire enfants, dans le sens métaphorique du terme, mais réellement de faire comme leurs propres enfants. Comme dans le Nouveau Testament, imiter l'enfant signifie pour l'homme adopter une attitude de simplicité face au Royaume de Dieu. Tout comme l'enfant, qui, par définition, est à la merci des adultes et n'a d'autre choix que de s'en remettre à eux, l'homme qui

93. "O vecchi, imparate da questi fanciulli a laudare il Signore», G. Savonarola, Prediche sopra Amos, ouvr. cité, XL, vol. 3, p. I55.

94. "[...] el Signore ha custodia de’ piccolini; e’ parvuli e piccolini sono quelli che si umiliano e abbassano e vanno semplicemente», G. Savonarola, Prediche sopra Aggeo, ouvr. cité, VII, p. II9.

95. Dictionnaire de la spiritualité, Paris, Beauchesne, 1960, vol. 4, p. 684-685 et p. 691.

96. "Se voi non diventate piccolini, non entrerete nel reame dé cieli; però datevi, dico, alla simplicità e alla umilità, ch'el Signore sarà con voi», G. Savonarola, Prediche sopra Aggeo, ouvr. cité, VII, p. II9. Voir Marc, IO, I3-I6; Matthieu, I9, I3-I5; Luc, I8, I5-I7.

97. G. Savonarola, Prediche sopra Amos, ouvr. cité, XIX, vol. 2, p. 43; ibid., XXXVI, p. I9-20; G. Savonarola, Prediche sopra Ruth, ouvr. cité, V, vol. I, p. IsI.

98. "[...] el bisogna diventare bambino, chi vuole entrare in vita eterna [...]", G. Savonarola, Prediche sopra l'Esodo, P. G. Ricci (éd.), Rome, Angelo Belardetti, VIII, vol. I, p. 225. 
veut entrer dans le Royaume n'a d'autre choix que d'avoir une attitude d'abandon et de confiance ${ }^{99}$. Entre le premier et le dernier cycle de sermons, l'enfance réelle a rejoint l'enfance métaphorique pour exprimer une seule et même idée : les fanciulli sont maintenant les guides de leurs pères, ils sont le modèle de chair et d'os que les adultes doivent imiter pour conduire leur cité au renouveau.

En moins de quatre ans, l'ordre traditionnel des générations est totalement inversé par Savonarole. Alors que les Florentins sont habitués à marcher sur les traces de leurs aînés, voilà qu'un prédicateur leur explique que seuls les fanciulli ont compris et respecté le projet de réforme qui plaît à Dieu et qu'il faut par conséquent suivre leur exemple, parce que les vieux qui se disent sages au nom de leur âge sont ridicules et que leur prétention à guider les jeunes n'est qu'abus de pouvoir et usurpation d'autorité. Dans le même temps, le fait que «l'expérience du bien vivre» soit gravée dans le sacrifice du fils de Dieu sur la croix fait dépendre les Florentins n'ont pas d'une expérience transmise de génération en génération, mais d'un enseignement universel et atemporel. Et comme cet enseignement, délivré par les premiers chrétiens, peut être imité par les individus de tout âge, même les plus jeunes peuvent être des sages, à l'instar des fanciulli del frate qui défilent en procession.

La prédication savonarolienne bouleverse ainsi suffisamment la hiérarchie des âges de la vie pour qu'à la figure du vieillard modèle se substitue, au début du $\mathrm{XVI}^{\mathrm{e}}$ siècle, la figure du jeune héros.

Nous sommes vraiment peu obligés envers nos ancêtres qui, détournant l'Italie de ses bons ordres, l'ont soumise à un gouvernement de prêtres et de marchands, et employant la milice mercenaire, l'ont conduite à l'ignominie présente et à l'esclavage ${ }^{100}$ !

Tels sont les termes qu'emploie Lodovico Alamanni, dans un texte écrit en I5I6, pour évoquer la présence des armées étrangères sur le sol italien. Or, de façon surprenante, ses invectives ne visent pas les soldats venus d'outre-monts. Pour Alamanni, les responsables de la situation désastreuse

99. Simon Légasse, Jésus et l'enfant. "Enfants», "petits» et "simples» dans la tradition synoptique, Paris, Librairie Lecoffre, 1969, p. 190-193, p. 36-40 et p. 230; Dictionnaire de spiritualité, ouvr. cité, vol. 4, p. 690-692. Ioo. "Pocho obligo habbiamo veramente co" nostri antichi, e quali, deviando Italia da' suoi buoni ordini, la ridussono ad governo di preti et di mercanti, et mettendo in uso la militia mercenaria, l'hanno condotta alla presente ignominia et servitù", Rudolf von Albertini (éd.), Lodovicus Alamannus, Ill. domino domino Alberto Pio, carpensi principi et cesareo oratori, S. D., dans Firenze dalla repubblica al principato. Storia e coscienza politica, Turin, Einaudi, 1995, p. 386. 
dans laquelle se trouve la péninsule ${ }^{\text {Ior }}$ sont en effet les générations qui ont précédé la sienne, parce qu'elles ont confié la défense militaire à des troupes mercenaires.

En réalité la polémique envers les soldats mercenaires n'est pas nouvelle et remonte même à l'époque des humanistes ${ }^{102}$, quand un Leonardo Bruni regrettait le temps de la commune médiévale où «le peuple florentin luimême descendait sur le terrain et prenait les $\operatorname{armes}^{103}{ }^{\prime}$. Car jusqu'à l'abolition en $135 \mathrm{I}$ du service militaire, qui concernait avant tout les citoyens aptes à occuper des charges publiques, le noyau des armées italiennes était constitué par les milices communales, les troupes mercenaires ne constituant qu'une force d'appoint ${ }^{104}$. De sorte que les citoyens défendaient leurs propres biens, leur propre indépendance, leur propre vie, comme l'explique Lodovico Alamanni :

Et sachant que s'ils ne gagnent pas, leurs femmes et leurs fils, leur patrie et leurs biens tomberont entièrement entre les mains de leur ennemi, ils sont obligés d'être courageux et constants; et ils voient que les dommages causés par la fuite sont si grands, que ceux de la mort leur semblent bien inférieurs. Et c'est pourquoi ils considèrent moins la vie, la plaçant après la victoire ${ }^{\mathrm{IOS}}$.

En revanche, les mercenaires ne sont motivés que par l'appât du gain ; aussi sont-ils dépourvus de cet esprit de sacrifice pourtant indispensable à la survie des États — on connaît à ce propos les observations de Machiavel au sujet des armes mercenaires, "inutiles et dangereuses" puisqu' «elles n'ont d'autre amour ni d'autre raison de rester aux armées qu'un peu de solde, qui ne suffit pas à faire qu' elles veuillent mourir pour toi ${ }^{106}{ }^{\prime}$.

IOI. Charles VIII est parvenu à Naples en six mois, sans rencontrer aucune résistance et en I5I6 un traité franco-espagnol a coupé l'Italie en deux, sous domination française au nord et sous domination espagnole au sud. Voir Ivan Cloulas, "La découverte de l'Italie par Charles VIII", dans J. Balsamo (dir.), Passer les Monts. Français en Italie, l'Italie en France (I494-I5225). Xe colloque de la Société française d'étude du Seizième Siècle, Paris, Fiesole, Honoré Champion, Cadmo, 1998, p. I27-138.

I02. Quentin Skinner, Le origini del pensiero politico moderno, Bologne, Il Mulino, I989, vol. I : Il Rinascimento, p. 153-154; Hans Baron, La crisi del primo Rinascimento italiano, Princeton, Princeton University Press, I966, p. $47 \mathrm{I}$.

I03. "[...] ipso populo Florentino exeunte atque armis fruente», cité par H. Baron, ibid., p. 469.

I04. Piero Pieri, Il Rinascimento e la crisi militare italiana, s.1. (Turin), Einaudi, 1952, p. 262-263. L'emploi des mercenaires se développe tout particulièrement à l'époque des guerres contre la Milan expansionniste des Visconti. G. Brucker, Dal Comune alla Signoria. La vita pubblica a Firenze nel primo Rinascimento, Bologne, Il Mulino, I98I, p. I45-238.

I05. "Et sapendo che, se non vincono, le lor moglie e lor figliuoli, la lor patria et la lor roba tutta viene in potestà del nimico, sono constretti ad essere animosi et constanti; et veggono tanto esser grande il danno della fuga, che quel della morte è molto minore. Et per questo sprezono la vita, posponendola alla victoria", R. Albertini, Firenze dalla repubblica al principato, ouvr. cité, p. 386.

I06. "inutile e pericolose", "[...] le non hanno altro amore né altra cagione che le tenga in campo che un pò di stipendio, il quale non è suffiziente a fare che vogliono morire per te», Machiavel, De principatibus - Le 
Cependant, au début du Xvi ${ }^{\mathrm{e}}$ siècle, la critique des troupes mercenaires prend la forme bien particulière d'un réquisitoire mené par les jeunes contre les vieux.

Il faut dire que pour la génération des Florentins qui ont grandi avec la guerre, Florence n’a jamais été bien gouvernée. En I522, Francesco Guicciardini, constatant que Florence n'a jamais connu la vraie liberté, entreprend de rédiger le Dialogo del reggimento di Firenze dans le but de montrer

comment on doit introduire à Florence un gouvernement honnête, bien ordonné, et qui pourrait vraiment être appelé libre, et ce alors même que depuis la première origine de la cité jusqu'à aujourd'hui, jamais aucun citoyen ne put ou ne sut le faire ${ }^{107}$.

Semblablement, Machiavel écrit après la mort de Lorenzo de’ Medici qu’à Florence "ne s'est jamais trouvé ni république ni principat ayant eu les qualités requises ${ }^{108}$ ». En filigrane se dessine la figure des vieilles générations, implicitement accusées d'incompétence politique.

Les vieux ont en effet été habitués à gouverner dans un contexte où après la paix de Lodi que les différentes puissances italiennes avaient signée en I454, la diplomatie pouvait dénouer les conflits. Or ces vieux se montrent incapables de prendre la mesure de la nouvelle "qualité des temps» introduite par les guerres d'Italie. Alors qu'on s'est mis, comme le relève Francesco Guicciardini, "à conserver, à abattre, à donner et à enlever les États non selon des desseins, non dans un cabinet - comme par le passé - mais sur le terrain, les armes à la main ${ }^{109}$ ", ils s'obstinent à gouverner avec le «bienfait du temps», se reposant sur l'attentisme et les manœuvres dilatoires. De plus, les vieux «sont incorrigibles", comme le relève Machiavel dans l'Arte della Guerra ${ }^{\text {IIO }}$. Incapables de se débarrasser du mauvais pli qu'ils ont pris, ils ne peuvent donc plus apparaître comme

Prince, G. Inglese (éd.), traduction et commentaire de J.-L. Fournel et J.-C. Zancarini, Paris, PUF, 20oo, XII, p. II6-II7.

I07. Francesco Guicciardini, Écrits politiques. Discours de Logrogno. Dialogue sur la façon de régir Florence, trad. J.-L. Fournel et J.-C. Zancarini, Paris, PUF, I997, p. I08. [" [...] come s'avessi a introdurre in Firenze uno governo onesto, bene ordinato, e che veramente si potessi chiamare libero; il che dalla sua prima origine insino a oggi non è mai stato cittadino alcuno che abbia saputo o potuto fare»], F. Guicciardini, Dialogo del Reggimento di Firenze, G. M. Anselmi et C. Varotti (éd.), Turin, Bollati Boringhieri, 1994, p. 15. Voir aussi F. Guicciardini, Le Cose fiorentine, R. Ridolfi (éd.), Florence, Leo S. Olschki, 1983, p. 2 I.

I08. "[...] in quella non è stato mai né repubblica né principato che abbi avute le debite qualità sue", Niccolò Machiavelli, Discursus florentinarum rerum post mortem iunioris Laurentii Medices, dans M. Martelli (éd.), Tutte le Opere, Florence, Sansoni, 1993, p. 24.

I09. "[...] a conservare, a rovinare, a dar ed a tôrre non co' disegni e nello scrittoio come pel passato, ma alla campagna e colle arme in mano", F. Guicciardini, Storie fiorentine, A. Montevecchi (éd.), Milan, BUR, I998, p. 197.

IIo. N. Machiavelli, Dell'Arte della guerra, dans Tutte le Opere, ouvr. cité, I, p. 305. 
l'incarnation de la sagesse politique, s'attirant aussi par là les critiques des jeunes gens.

Savonarole expliquait que les Florentins avaient perdu leur "première forme" à cause de l'enseignement que les aînés s'obstinaient à transmettre à leurs descendants. Pour protéger la cité des tribulations, il fallait par conséquent que les Florentins renoncent au "vieil usage» (la sagesse usurpée des vieux) pour instaurer une «nouvelle coutume» (la réforme voulue par Dieu et défendue par les fanciulli) qui leur permettrait de retrouver leur "première forme" (les vertus des premiers chrétiens et des saints). Semblablement, Lodovico Alamanni espère que Florence saura abandonner la mauvaise habitude qui est la sienne (l'emploi des troupes mercenaires) pour imposer la «bonne coutume» (les troupes de citoyens-soldats) de façon à retrouver «son lit ordinaire» (la force militaire du temps de la Commune). Il écrit ainsi qu'

il faut avec la bonne coutume chasser la mauvaise [...] parce que le fleuve dont le cours a été changé de force, même s'il est resté ainsi pendant longtemps, retourne néanmoins toujours facilement dans son lit ordinaire ${ }^{\mathrm{III}}$.

Car la faiblesse politique et militaire des Florentins, explique-t-il, «vient de la mauvaise coutume et non de leur nature faible ${ }^{\mathrm{II} 2}$ ». L'argument des "vieux [...] [qui] disent que la nécessité pousse les Florentins à employer les troupes mercenaires parce que leurs peuples ne sont pas aptes à porter les armes ${ }^{\mathrm{II}}{ }^{\text {» }}$ est donc selon lui fallacieux. Descendants des Étrusques, les Florentins sont des guerriers, de sorte que l'art de se battre fait partie d'eux-mêmes. Et puisque l'incapacité des Florentins à manier les armes résulte d'un processus qui relève de la coutume, il faut leur faire perdre les mauvaises habitudes données par les vieux, de façon à leur redonner l'essence militaire qui leur est propre.

Lorsqu'il évoque le passé étrusque de Florence, Alamanni se tourne vers une époque ancienne, une époque qui apparaît exemplaire par opposition à l'histoire récente de Florence, comme les martyrs et les saints étaient destinés, chez Savonarole, à effacer les mauvais plis que les Florentins se donnaient de génération en génération. Machiavel procède de façon semblable lorsqu'il offre aux lecteurs des Istorie fiorentine des exemples à fuir en même temps que des modèles à imiter :

III. "Bisognia dunche con la buona consuetudine cacciar la trista [...] perché quel fume ad chi per forza fu fatto mutare il corso, benché così sia ito un pezzo, nondimeno sempre facilmente si ritorna al suo letto ordinario [...]», R. Albertini, Firenze dalla repubblica al principato, ouvr. cité, p. 387.

II2. "[...] viene da la mala consuetudine et non da la debile loro natura", ibid., p. 386.

II3. "[...] vecchi [...] dicono che la necessità induce e fiorentini ad usar le milite mercennarie perché e loro popoli non sono atti al'arme [...]", ibid., p. 386. 
l'histoire récente ne sera peut-être pas moins utile que la connaissance de l'histoire ancienne, car, si cette dernière pousse les esprits libres à l'imiter, l'autre les encouragera à fuir et à nier ces autres comportements ${ }^{114}$.

Puisque les exemples les plus récents appartiennent au temps où la cité n’a jamais été bien gouvernée, ils servent en effet à montrer ce qu'il ne faut pas faire : les hommes qui ont mal gouverné la cité ne sauraient être des modèles à imiter. À l'inverse, Machiavel se tourne vers l'histoire romaine qui contient des figures politiques exemplaires.

Car les Romains possèdent trois qualités que n’ont pas les Florentins. Ils savaient anticiper les difficultés, "voir de loin", au lieu de se délecter d'une attitude attentiste et dilatoire. On connaît à ce propos le passage du chapitre III du Prince, où Machiavel explique que les Romains

firent dans ces cas-là ce que tous les princes sages doivent faire : ceux-ci ne doivent pas seulement prendre garde aux scandales présents, mais aussi aux futurs, et mettre toute leur industrie à y obvier [...] Et jamais ne leur plut ce qu'ont chaque jour à la bouche les sages de notre temps, "il faut jouir des bienfaits du temps ${ }^{\mathrm{II}}$.

Les Romains avaient aussi mis en place une pédagogie militaire efficace, formant les jeunes au combat et tenant les vieux à l'écart. Sur ce point, les Romains sont donc aussi à imiter puisqu'

ils se prévalaient des hommes dans la fleur de l'âge, depuis dix-huit jusqu'à trentecinq ans, âge où les jambes, les mains et l'œil répondaient les uns aux autres; et ils n'attendaient pas que leurs forces diminuent et que leur malice augmente ${ }^{\mathrm{I1}}$.

Enfin, Machiavel explique combien les Romains conduisaient la guerre de façon pertinente :

en finissant les guerres promptement, tout en étant capables d'épuiser leurs ennemis sur le long terme, par des défaites, des incursions et des accords à leur avantage, ils devinrent toujours plus riches et plus puissants ${ }^{117}$.

\footnotetext{
II4. "[...] sarà forse non meno utile che si sieno le antiche cose a conoscere, perché, se quelle i liberali animi a seguitarle accendono, queste a fuggirle e spegnerle gli accenderanno", N. Machiavelli, Istorie fiorentine, dans Tutte le Opere, ouvr. cité, V, I, p. 739.

II5. "[...] feciono in questi casi quello che tutti e principi savi debbono fare: li quali non solamente hanno ad avere riguardo alli scandali presenti, ma a' futuri, et a quelli con ogni industria obviare [...] Né piacque mai loro quello che è tutto dì in bocca de' savi de' nostri tempi, di godere il benefizio del tempo", Machiavel, De principatibus, ouvr. cité, III, p. 54-57.

II6. "[...] si valevano degli uomini nel fiore della loro età, che è da' diciotto a' trentacinque anni, nel qual tempo le gambe, le mani e l'occhio rispondevano l'uno all'altro; nè aspettavano che in loro scemasse le forze e crescesse la malizia», N. Machiavelli, Dell'Arte della guerra, ouvr. cité, p. 537.

II7. "[...] con il finire le guerre presto, sendo valenti con lunghezza straccare $i$ nemici, e con le rotte e con le scorrerie e con accordi a loro vantaggi, diventarono sempre più ricchi e pù potenti", N. Machiavelli, Discorsi sopra la prima deca di Tito Livio, G. Inglese (éd.), Milan, Rizzoli, I996, II, 6, p. 3II.
} 
Cette façon de faire la guerre correspondant aux exigences de la nouvelle "qualité des temps» quand les Français, comme le dit Francesco Guicciardini, "introduisirent dans la guerre une grande rapidité ${ }^{118}$ ", les Romains constituent pour les Florentins des modèles à imiter.

Cependant, si pour les marchands florentins l'autorité des ancêtres ne faisait aucun doute et si pour Savonarole les premiers chrétiens incarnaient le bien de façon absolue, Machiavel ne considère pas que l'exemple des Romains est en soi meilleur que d'autres. Comme on le sait, le passé doit être en permanence confronté à l'expérience contemporaine, qui recouvre une fréquentation quotidienne des problèmes liés à l'organisation de la cité. Car il s'agit moins de reproduire l'histoire que de la comprendre grâce à l'expérience du présent. Voilà pourquoi l'imitation n'est pas une fin en soi, mais uniquement un moyen par lequel l'homme prudent parvient à agir. Dans une société qui fait de l'imitation de modèles le centre de toute éducation - et Savonarole l'avait parfaitement compris -, Machiavel ne voit donc que des exemples qui doivent nourrir la réflexion.

De sorte que pour la génération de la guerre, dont Machiavel est emblématique, l'éducation du citoyen devra désormais reposer davantage sur le dépassement des générations précédentes que sur la reproduction imitative de leurs actes.

L'exemple de la famille Médicis est une fois de plus significatif. Au $\mathrm{XV}^{\mathrm{e}}$ siècle, les citoyens influents de Florence avaient choisi Laurent le Magnifique pour sa capacité supposée à marcher sur les traces de ses ancêtres et à en être digne. Le jeune dirigeant souligne lui-même dans son livre de famille qu'il est parvenu à bien gouverner non pas grâce à sa prudence, mais grâce «à la bonne conduite de $[\mathrm{s}]$ es ancêtres ${ }^{\mathrm{I}{ }^{19}}$ ». Deux décennies plus tard, un membre de la chancellerie écrit semblablement à propos de Piero de' Medici que «tous s'accordaient à lui faire honneur, parce qu'ils espéraient qu'il serait un excellent héritier de toutes les vertus de son père ${ }^{120}$ ", et on se souvient que la déception que ressentent les Florentins sur ce point lui vaut d'être chassé de la cité en I494.

Mais au début du XVI ${ }^{\mathrm{e}}$ siècle, dans une société où l'autorité des ancêtres n'est plus incontestable et incontestée, Piero est excusé pour avoir failli à

II8. "[...] introdussono nelle guerre tanta vivezzza [...]», F. Guicciardini, Ricordi, E. Pasquini (éd.), Milan, Garzanti, 1988, LXIV, p. 85.

II9. "[...] per li buoni portamenti de’ [m]iei passati», Lorenzo de’ Medici, Opere, T. Zanato (éd.), Turin, Einaudi, I992, p. xxxix.

I2O. "[...] tutti a una s'accordono a farli onore, sperando che lui abbia a essere optimo herede paterno di tutte le sue virtù", cité par Nicolai Rubinstein, Il governo di Firenze sotto i Medici (I434-I494), Florence, "La Nuova Italia» Editrice, I97I, p. 279-280. 
son devoir de répétition. Alessandro de’ Pazzi explique ainsi que le jeune Médicis a perdu le pouvoir

non pas tant parce qu'il n'avait pas la même intelligence excellente et sublime [que son père], mais surtout parce que la puissance française vint en Italie, et que le roi Charles perturba toutes les choses en y passant ${ }^{121}$.

Dans un contexte où la guerre est désormais le seul horizon, les exemples à reproduire ne sont plus incarnés par les vieux qui s'en remettaient à la voie diplomatique, mais par des jeunes gens fougueux qui n'hésitent pas à prendre les armes, à l'instar de Paolo Vettori et de ses compagnons qui en 1512 ont chassé le gonfalonier de justice Piero Soderini par la force ${ }^{\mathrm{I} 22}$. Voilà pourquoi ce même Paolo Vettori peut recommander aux Médicis de ne surtout pas imiter la façon dont leurs aïeux ont gouverné Florence :

Vos ancêtres, à commencer par Côme et jusqu’à Piero, ont employé davantage la ruse que la force pour dominer cet État. Mais pour vous il est nécessaire d'utiliser davantage la force que la ruse ${ }^{123}$.

Du reste, la génération de la guerre défend l'idée selon laquelle la compétence politique n'est plus déterminée par la valeur des ancêtres, mais par celle des individus. Chacun doit faire la preuve de son propre mérite, comme Bernardo del Nero, que Francesco Guicciardini met en scène dans le Dialogo del Reggimento di Firenze, et qui a gravi tous les échelons du cursus honorum alors qu'il n'était ni "d'un noble lignage ni entouré de parents $^{124} »$. Il faut en finir, précise Guicciardini, avec la coutume qui fait que «jusqu'au jour d'aujourd'hui [...] celui qui n’est pas né en étant pourvu de la réputation de ses pères et de sa maison n'a eu aucune possibilité de se faire connaître aisément ${ }^{125}$ ». Pour Machiavel, le pouvoir transmis de façon héréditaire est même source de décadence : comme les héritiers n'ont pas eu besoin d'agir pour acquérir leur position, ils ne cherchent

\footnotetext{
I2I. "[...] non tanto perché non fussi di quella eccellenza e sublimità d'ingegno; ma ancora perchè la potenza francese venne in Italia, e il re Carlo perturbò tutte le cose sue con la passata sua in essa", Discorso di Alessandro de' Pazzi, al Cardinale Giulio de’ Medici, anno I522, G. Capponi (éd.), Archivio Storico Italiano, I842, t. I, p. 423. I22. Sur cet épisode, R. Albertini, Firenze dalla repubblica al principato, ouvr. cité, p. 2I-22 et Giovanni Silvano, "Vivere civile» e "governo misto» a Firenze nel primo Cinquecento, Bologne, Pàtron, 1985, p. 24-25.

I23. "Li antecessori vostri, cominciandosi da Cosimo e venendo infino a Piero, usorno in tenere questo Stato piu industria che forza. A voi è necessario usare più forza che industria, perché voi ci avete più nimici e manco ordine a saddisfarli", Ricordi di Paolo Vettori al cardinale de' Medici sopra le cose di Firenze, dans R. Albertini, Firenze dalla repubblica al principato, ouvr. cité, p. 357.

I24. "[... non essendo io di stirpe nobile, né cinto di parenti», F. Guicciardini, Dialogo del reggimento, ouvr. cité, p. 26.

I25. "[...] insino a oggi [...] chi non è nato con la riputazione de’ padri o della casa, non ha avuto facultà facile di farsi conoscere", ibid., p. I82.
} 
qu’à jouir de ce que leur ont légué leurs ancêtres, sans chercher à exercer leur propre vertu pour se maintenir au pouvoir ${ }^{\mathrm{12}}$.

L'expérience transmise par les ancêtres ne suffit donc plus à définir la compétence du citoyen. L'individu ne s'inscrit plus dès lors dans l'histoire collective de ses aïeux, mais dans sa propre expérience de l'histoire. De ce point de vue, la diversité des écrits de Francesco Guicciardini est significative. Là où les marchands florentins rassemblaient en un seul livre le récit de la geste ancestrale, l'histoire publique de la cité ainsi que des bribes d'autobiographie, Guicciardini opère une séparation très nette. Alors qu'il garde jalousement, dans le secret des Memorie di famiglia, l'histoire de ses ancêtres qu'il glorifie, il la réutilise dans ses Ricordi comme un outil de réflexion politique. Quant à l'histoire de la cité, si elle est racontée dans les Memorie pour glorifier la famille Guicciardini, elle sert dans les Storie fiorentine à participer au débat politique. Pour former le citoyen à travers une expérience vécue et non une expérience héritée des ancêtres, l'histoire publique doit donc être écrite en dehors de l'histoire privée. Voilà aussi pourquoi Guicciardini consacre les Ricordanze à raconter sa propre carrière, comme pour signifier qu'il ne la doit qu'à lui-même et non à l'enseignement qu'il a reçu de ses aïeux ${ }^{\mathrm{I} 27}$.

Les ancêtres ne sont donc plus des figures d'autorité, non seulement parce que les stratégies politiques qu'ils défendent, telles que le «bienfait du temps", ne semblent plus adaptées à la nouvelle conjoncture historique, mais aussi parce qu'il apparaît nécessaire que les jeunes gens se forment, par leur propre pratique, à la politique, sans se contenter d'une expérience héritée. Or, en même temps que les vieux perdent leur fonction de modèles, une nouvelle figure émerge : celle du jeune héros.

$\mathrm{Au}$ début du $\mathrm{Xv}^{\mathrm{e}}$ siècle, Coluccio Salutati écrivait :

Il y a bien plus d'enfants, bien plus de jeunes tombés au combat que de vieillards, et ceux que leurs cheveux blancs rendent vénérables sont moins nombreux à périr dans les incendies ou écrasés sous les ruines que ceux qui sont redoutables par leur force ou pitoyables étant dans les prémices de leur âge ${ }^{\text {I28 }}$.

I26. N. Machiavelli, Discorsi, ouvr. cité, III, 34.

I27. C. Terreaux-Scotto, «Histoire de la famille et histoire de la cité chez Francesco Guicciardini», dans J.-J. Marchand, et J.-C. Zancarini (dir.), Storiografia repubblicana fiorentina (I494-I570), Florence, Franco Cesati Editore, 2003, p. 5I-70.

I28. "Longe plures pueri pluresque iuvenes ceciderunt bello quam senes paucioresque absumpserunt incendia appresseruntque ruine venerandos canicie quam robore metuendos vel etatis primiciis lacrimando", Coluccio Salutati, Epistolario, F. Novati (éd.), Rome, Forzani e C. Tipografi del senato, I89I, vol. I, p. 315. 
La sagesse apparaissait ainsi comme la meilleure protectrice des vieillards, y compris contre la faiblesse physique qui lui est associée depuis Homère' ${ }^{129}$ ! Aussi l'ancêtre idéal que les jeunes gens étaient invités à contempler et à imiter dans les livres de famille était-il un vieux sage doté d'une force exceptionnelle, à l'image d'un Ruzza Niccolini ou d'un Bonaccorso Velluti. Mais d'une part, la prédication savonarolienne a montré combien la sagesse pouvait ne pas dépendre du nombre des années; d'autre part, les nécessités de la guerre ont montré combien la force, et non la prudence précautionneuse, devenait le rouage essentiel de la vie politique. Dans ces conditions, la seule défense efficace en cas d'attaque sera, comme le recommande Machiavel dans l'Arte della guerra, "que les femmes, les vieux, les enfants et les faibles restent chez eux et laissent le terrain libre aux hommes jeunes et robustes ${ }^{130}$ ", parce que ces derniers sont "plus prompts à $[. .$. mettre en pratique» «l'exercice militaire ${ }^{\mathrm{I3I}} »$.

L'archétype du soldat incapable sera alors un Luca Savelli, qui, envoyé à Prato en I5I2, échouera lamentablement dans sa mission et que Francesco Guicciardini qualifie de "vieux condottiere mais à qui ni l'âge ni l'expérience n'avaient permis d'atteindre le moindre degré de connaissance dans la science militaire ${ }^{\mathrm{I32}}$ ». À l'inverse, Gaston de Foix est à vingt-trois ans l'incarnation du jeune soldat vaillant "puisque — déjà capitaine avant même d'avoir été soldat — il avait, en moins de trois mois, remporté tant de victoires avec une rapidité et une fougue étonnantes ${ }^{133}$ ». Le soldat idéal n'est toutefois pas seulement fort. Il est aussi sage, alliant le courage de l'homme jeune à la prudence du vieillard, tel le duc de Bourbon, dont Guicciardini écrit qu' «encore jeune - il n'avait pas trente-six ans

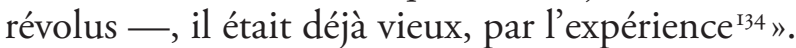

Le soldat modèle apparaît ainsi comme le double inversé des héros que les Florentins avaient jusqu'alors exaltés dans leurs livres de famille : des

I29. Homère, Illiade, trad. Paul Mazon, Paris, "Les Belles Lettres», I964, t. 4, XXIII, v. 590 et 604. Voir Ernst Robert Curtius, La littérature européenne et le Moyen Âge latin, Paris, PUF, 1956, vol. I, p. 283-288.

I30. "[...] che le donne, $i$ vecchi, $i$ fanciulli e $i$ deboli si stieno in casa e lascino la terra libera a' giovani e gagliardi», N. Machiavelli, Dell'Arte della guerra, ouvr. cité, VII, p. 38I. Voir aussi ID., Discorsi..., II, 29, p. 373. I3I. "più pronti a esequirlo", "l'esercizio", N. Machiavelli, Dell'Arte della guerra, ouvr. cité, III, p. 334.

132. "[...] condottiere vecchio ma che né per l'età né per l'esperienza era pervenuto a grado alcuno di scienza militare», F. Guicciardini, Storia d'Italia, E. Mazzali (éd.), Milan, Garzanti, I988, XI, 3, vol. 2, p. II8I. La traduction, comme celle d'autres extraits de la Storia d'Italia, provient de : F. Guicciardini, Histoire d'Italie I492-I534, traduction de l'italien dans le cadre de l'atelier de traduction du Centre de recherche sur la Pensée politique italienne de l'ENS de Fontenay-Saint-Cloud sous la direction de J.-L. Fournel et J.-C. Zancarini, Paris, Robert Laffont, 1996.

133. "[...] avendo in manco di tre mesi, e prima quasi capitano che soldato, con incredibile celerità e ferocia ottenuto tante vittorie», ibid., X, I3, vol. 2, p. II3I.

134. "[...] giovane di età, che non passava trentasei anni, era già vecchio di esperienza", ibid., XVI, II, vol. 3, p. 1839 . 
jeunes à la sagesse exceptionnelle se substituent aux vieux à la force extraordinaire. Désormais, le salut de la cité se trouve dans un soldat qui allie la force d'un Achille à la sagesse d'un Nestor. Castruccio Castracani, le héros que Machiavel propose comme modèle à Zanobi Buondelmonti et Luigi Alamanni, est de ceux-là, puer maior sua etate qui, dès qu' «il commença à étudier le métier des armes» à l'âge de quatorze ans,

ne prenait plaisir qu’à leur maniement ou à courir avec ses camarades, à sauter, à lutter et à d'autres exercices semblables. Il y montrait une très grande vertu de corps et d'âme, supérieure à celle de tous les garçons de son âge ${ }^{135}$.

Aux ancêtres, que les jeunes gens étaient censés imiter jusqu'à ce que Savonarole charge les fanciulli de montrer le bon exemple à leurs aînés, succèdent dès lors les soldats, pueri senes ${ }^{136}$ qui défendent la République florentine non plus par la correction fraternelle, mais les armes à la main, en particulier au moment où la cité est menacée, puis assiégée, par les troupes espagnoles entre 1529 et 1530 .

Le 6 novembre I528, la défense de Florence a en effet été confiée aux Florentins, et plus exactement aux jeunes de moins de trente-six ans, qui seront encouragés dans leurs efforts à l'occasion de harangues prononcées une fois par an dans l'église principale de chacun des quatre quartiers ${ }^{137}$.

Pour les orateurs, ces dispositions montrent que les hommes nés avec le siècle sont désormais clairvoyants; délivrés de leur myopie, ils se sont débarrassés de la coutume du commerce qui leur avait fait perdre leur vraie nature, c'est-à-dire la pratique des armes. Luigi Alamanni considère à ce propos que

Dieu en voulant nous montrer un si grand bien, et que nous le connaissions, n'a pas pu trouver de façon plus belle ni plus sûre que d'enlever de nos yeux le brouillard des richesses, et de nous mettre sur les collines de la pauvreté et de l'indigence : d'où nous pouvons discerner plus ouvertement ce que nos ancêtres ne virent jamais ${ }^{138 .}$

\footnotetext{
135. «[...] cominciò a trattare le armi; né di altro si dilettava che o di maneggiare quello, o con gli altri suoi equali correre, saltare, fare alle braccia, e simili esercizii; dove ei mostrava virtù di animo e di corpo grandissima, e di lunga tutti gli altri della sua età superava", N. Machiavelli, La vita di Castruccio Castracani da Lucca descritta da Niccolò Machiavelli e mandata a Zanobi Buondelmonti e a Luigi Alamanni suoi amicissimi, dans Tutte le Opere, ouvr. cité, p. 616.

I36. Voir à ce propos Frédérique Verrier, Les armes de Minerve. L'Humanisme militaire dans l'Italie du XVI siècle, Paris, Presses de l'Université de Paris-Sorbonne, 1997, p. I54.

137. Sur toute la question des harangues, je renvoie à la contribution de Silvia Genzano dans ce même volume. 138. «[...] volendo Dio mostrarci un tanto bene, e che noi lo conoscessimo, non ha potuto trovar modo più bello e più sicuro che levarci davanti agli occhi la nebbia delle ricchezze, e metterci sopra i colli della povertà e della necessità: onde più apertamente potessimo scernere quello che i nostri antichi non vider mai», Luigi Alamanni, Orazione al popol fiorentino sopra la militar disciplina, dans Versi e prose, P. Raffaelli (éd.), Florence, Felice Le Monnier, I859, vol. 2, p. 450.
} 
Les jeunes, qui ont été capables de rejeter les mauvaises habitudes que leur avaient inculquées leurs aînés, ont ainsi pu redonner sa vraie «nature» à Florence, celle d'une cité guerrière à l'image d'Athènes, de Sparte ou de Rome dont elle a imité «les merveilleux ordres salvateurs ${ }^{139}$ ». Florence peut donc retrouver la gloire des armes parce que, comme le dit Piero Vettori,

nous sommes bien nés sous le même ciel capable de produire des âmes généreuses et splendides, et il ne faut pas estimer que la nature a changé, mais que la cause de notre sommeil paresseux a été plutôt la mauvaise éducation qui anéantit et endort les esprits généreux en les nourrissant d'œuvres abjectes ${ }^{140}$.

Comme l'avait souhaité Savonarole, les jeunes n'imitent donc plus leurs ancêtres. Bien plus, de la même façon que les fanciulli del frate dépassaient leurs aînés en sagesse, les jeunes de la milice incarnent «l'antique valeur" italienne célébrée par Pétrarque et que Machiavel souhaitait voir renaître grâce à Laurent de Médicis. Piero Vettori encore se réjouit en ces termes :

Ô Dieu glorieux et très haut, qui as toujours pris un soin particulier de ta cité, on voit bien clairement que tu ne veux pas entièrement détruire ce malheureux peuple, puisque tu as ravivé dans les poitrines de ces valeureux soldats cette antique vertu, qui a été longtemps cachée et ensevelie. C'est bien le signe que pourrait ressusciter l'antique valeur qui guérirait ton pays aimé des blessures mortelles qu’on voit en lui et qui le libérerait des plaies honteuses de l'esclavage, puisque tu as engendré dans cette belle jeunesse des âmes si sincères et des cours si résolus qu'ils se jettent si hardiment dans tous les dangers pour sauver notre douce liberté et déverser une juste colère contre les ennemis ${ }^{141}$.

Par ailleurs, Filippo Parenti explique comment les Florentins, favorisés à la fois par leur «disposition naturelle» et par la "disposition céleste», et qui possèdent donc aussi bien le courage des habitants des régions froides

139. "maravigliosi e salutari ordini", Orazione di Bartolomeo Cavalcanti patrizio fiorentino fatta alla militare ordinanza fiorentina il di III febraio MDXXVIIII (stile comune I530) in Firenze, dans Orazioni scelte del secolo XVI, G. Lisio (éd.), Florence, Sansoni, 1957, p. I5.

I40. "[...] siamo pur nati sotto il medesimo cielo atto a produrre generosi animi et leggiadri, né s'ha a stimare ch'habbia mutata natura, ma che sia più tosto stato cagione del nostro pigro sonno la mala educatione channighittisce et addormenta ogni generoso spirito col nutrirlo in opere abiette", Orazione di Piero Vettori, fatta alla militare ordinanza fiorentina l'anno M.D.XXIX il dì (s febbraio), R. Albertini (éd.), dans ID., Firenze dalla repubblica al principato, ouvr. cité, p. 419.

I4I. "O glorioso et sommo Dio, c'hai sempre tenuta particular cura di questa tua città e' si vede pur chiaro che tu non vuoi interamente distrugger questo misero popolo, poi che tu hai raccesa ne' petti di questi valenti soldati quell'antica virtù, stata tanto tempo ascosa et sepolta. Quest'è pur segno ch'ancor potrebbe risurger quell'antico valore che sanasse questo tuo diletto paese delle mortali ferite che in lui si veggono et lo liberassi dalle vergognose piaghe di servitù, poi ch'hai generati si franchi animi et si resoluti cuori in questa bella gioventù che si arditamente e' si gettino in ogni pericolo per salvar la nostra dolce libertà et sfogare una giusta ira contro ai nemici", ibid., p. 42 I. 
que l'intelligence des habitants des régions chaudes, sont «les meilleurs soldats du monde $»^{\mathrm{I} 42}$.

Il n'y a pas seulement là une réhabilitation de la soldatesque, traditionnellement fustigée pour ses défauts — en I498, les Dix de guerre écrivaient par exemple à leur commissaire que «tous les soldats sont plutôt tournés à mal faire qu'à toute autre bonne opération ${ }^{143}{ }^{14}$. Car la célébration des jeunes gardiens de la République florentine est de l'ordre du comparatif de supériorité qui fait d'eux des héros.

Les jeunes gens sont en effet libérés de toutes les contraintes humaines, puisque leurs corps supportent sans faillir le manque de sommeil, la faim, la soif, la chaleur, le froid ${ }^{\mathrm{I} 4}$. Ces remarques de Cavalcanti sont d'autant plus significatives qu'en réalité ces jeunes gens n'ont pas eu à affronter de réels combats, les batailles étant encore le plus souvent livrées par des mercenaires à la solde de Florence ${ }^{\mathrm{I} 45}$. Pourtant, dans la bouche des orateurs, leur seule présence épargne la vie de leurs parents, de leurs enfants, de leurs femmes. C'est qu'il s'agit de galvaniser la cité en la confiant à des êtres supérieurs qui apparaissent comme de véritables sauveurs - ce rôle étant, comme l'a souligné Charles Baudoin — « un caractère éminent du héros" ${ }^{\mathrm{I} 46}$.

Mais ces jeunes Florentins n'accomplissent pas seulement des exploits extraordinaires pour sauver la ville élue. Car leur sacrifice pare ces héros épiques d'une dimension chrétienne ${ }^{\mathrm{I} 47}$. Véritables rédempteurs, ils donnent leur vie pour la liberté de Florence au point de porter les stigmates et d'apparaître dans un corps glorifié, comme le proclame un Bartolomeo Cavalcanti exalté :

\footnotetext{
I42. "disposizion naturale», "disposizione celeste», "i migliori soldati di tutto il mondo", Orazione di Filippo Parenti a' soldati della nuova milizia fiorentina, dans P. Dazzi (éd.), Orazioni politiche del secolo XVI, Florence, G. Barbèra, I866, p. 44I. Parenti s'inspire d'Aristote. Aristote, Les politiques, trad. P. Pellegrin, Paris, Flammarion, 1993, VII, 7, I327a-I327b. Sur l'influence déterministe du climat sur les hommes, Mario Pinna, La teoria dei climi. Una falsa dottrina che non muta da Ippocrate a Hegel, Rome, Società Geografica Italiana, I988.

I43. "tucti e' soldati son più tosto volti al far male che ad altra buona operatione", N. Machiavelli, Legazioni. Commissarie. Scritti di governo, F. Chiappelli (éd.), Bari, Laterza \& Figli, I97I-I973-1984-1985, vol. I, p. 8-9.

144. Orazione di Bartolomeo Cavalcanti, ouvr. cité, p. I7-18.

145. P. Pieri, Il Rinascimento e la crisi militare italiana, ouvr. cité, p. 5IO et 516.

146. Charles Baudoin, Le triomphe du héros. Étude psychanalytique sur le mythe du héros et les grandes épopées, Paris, Plon, 1952.

I47. Voir à ce propos Bernard F. Huppé, The Concept of the Hero in the Early Middle Ages, dans Norman T. Burns et Christopher Reagan, Concepts of the Hero in the Middle Ages and the Renaissance, Londres, Sydney, Auckland, Toronto, Hodder and Stoughton, 1975, p. I-26.
} 
On verra dans le ciel vos plaies resplendir de la lumière de la gloire divine, parce que, ayant empli le monde de la renommée du sang répandu pour le peuple élu de JésusChrist, celui-ci vous emplira de la béatitude éternelle ${ }^{\mathrm{I} 48}$.

\section{Piero Vettori insiste en prévenant les Florentins qu’}

on doit d'autant plus chérir ceux qui ont cette mort fortunée et glorieuse pour la patrie, qu'il leur est préparé au ciel un siège au milieu des esprits bienheureux où ensuite ils se réjouiront éternellement en contemplant le visage de leur Rédempteur ${ }^{149}$,

\section{tout comme Pandolfini qui promet que}

ceux qui rendent leur dernier souffle au service de la patrie, obtiennent dans le Ciel le siège des Bienheureux; et bien que leur vie ait été brève, la mémoire d'avoir vécu et d'être mort victorieusement est éternelle ${ }^{\mathrm{I} 5 \mathrm{O}}$.

C'est pourquoi ce dernier invite à rendre aux soldats de la milice un véritable culte post-mortem, parce qu'ils deviennent, comme tous les héros et les saints, des morts exceptionnels ${ }^{\mathrm{ISI}}$ : les vivants

feront en sorte que par la mémoire, par des statues, des tombeaux publics, qui conviennent aux œuvres louables, ceux qui auront été excellents dans la milice obtiennent la vie immortelle à la place de leur condition mortelle ${ }^{152}$.

Tout se passe donc comme si au cours des dernières années de la République florentine, les promesses savonaroliennes s'accomplissaient. Ce sont les jeunes générations qui sauvent Florence de l'entêtement des vieux. Non seulement l'ordre gérontocratique de la cité est totalement remis en cause, mais il est même inversé, puisque ce sont les jeunes soldats, héritiers des fanciulli del frate, qui sont des héros, alors que les vieux constituent au contraire les exemples à fuir.

\footnotetext{
148. "Vedransi in cielo le vostre piaghe lampeggiare della luce della divina gloria, perché, avendo noi ripieno il mondo della fama dello sparso sangue per lo eletto popolo di Jesù Cristo, egli di quella sempiterna beatitudine voi riempierà", Orazione di Bartolomeo Cavalcanti, ouvr. cité, p. 33.

I49. "[...] quanto più s'ha a haver caro ch'a quelli che fanno questa fortunata et gloriosa morte per la patria, è loro apparecchiata in cielo la sedia fra gli spiriti beati dove poi e' si godono eternamente la faccia dal lor Redentore", Orazione di Piero Vettori, ouvr. cité, p. 423.

I50. "[...] quelli che in servizio della patria mandano fuori l'ultimo spirito, conseguiscono in Cielo la sede de' Beati; et benchè la lor vita sia stata breve, la memoria d'esser vissuti et morti vittoriosamente è sempiterna", G. Canestrini (éd.), Oratione di Pier Filippo di Alessandro Pandolfini al Popolo di Firenze nel tempio di San Lorenzo, a dì XXVIII di gennaio MDXXVIII, "Documenti per servire alla storia della milizia italiana dal XIII secolo al XVI ", Archivio Storico Italiano, s. VI, t. XV, I85I, p. 373.

I5I. Sur ce point, J. Le Goff, Héros du Moyen Âge, le Saint et le Roi, ouvr. cité., p. I6-I7.

I52. "[...] con memoria di statue, di sepolcri publici, convenienti a l'opere laudabili, faranno che quelli che saranno stati eccellenti nella milizia, in cambio di condizione mortale conseguino vita immortale», G. Canestrini, art. cité, p. 374 .
} 
En conclusion, l'étude des «modèles» et des «héros» dans la Florence républicaine montre que chaque période historique est marquée par des références qui lui sont propres. Lorsque la prudence est la valeur maîtresse de l'art de gouverner, ce sont les vieillards, incarnations de la sagesse, qui sont présentés comme des modèles à suivre. Mais à partir du moment où les guerres d'Italie imposent d'envisager le recours à la force, ce sont les jeunes gens qui apparaissent comme les sauveurs de la cité, d'autant que le prédicateur dominicain Jérôme Savonarole a inversé, pendant près de quatre ans, l'ordre traditionnel des générations, qui soumettait les jeunes aux vieux.

Autant la notion de «modèle» est stable et renvoie à un type particulier d'éducation centrée autour de l'imitation et de l'exemplarité, autant la notion de "héros» suit les variations de l'histoire. Plus exactement, s'il est possible de définir à grands traits ce qu'est un héros (un être supérieur aux autres), on pourrait dire qu'il y a autant de héros que de contextes historiques. Le héros florentin des marchands $\mathrm{du} \mathrm{Xv}^{\mathrm{e}}$ siècle est un ancêtre prudent; mais les héros de la prédication savonarolienne sont les enfants et les jeunes adolescents qui imitent les premiers chrétiens et les martyrs; quant au héros de la dernière république florentine, il est un soldat fort et avisé, qui ne s'inscrit pas dans la continuité des générations familiales.

C'est que la vertu d'un individu se mesure désormais non pas à l'aune de la réputation de ses ancêtres, mais en fonction des actions glorieuses qu'il aura pu lui-même accomplir. L'artiste Benvenuto Cellini, qui a brièvement fait partie de la milice florentine avant de retourner à Rome ${ }^{153}$, est emblématique de cette évolution ${ }^{154}$. Rédacteur, dans la seconde moitié du XVI ${ }^{\mathrm{e}}$ siècle, de ce qui est considéré comme la première autobiographie moderne ${ }^{155}$, Cellini souligne d'emblée que chacun doit se conformer aux usages de la société dans laquelle il vit. Parmi les usages de la société florentine, il cite l'habitude marchande de "faire savoir aux autres que l'homme descend de personnes vertueuses et très antiques ${ }^{156}{ }^{15}$. Cependant, même s'il s'attribue une généalogie prestigieuse aux allures de légende (un ancêtre romain Fiorinus aurait même donné son nom à la ville de Florence!), il ne centre pas son récit autour des exploits accomplis par ses

153. Benvenuto Cellini, Vita, E. Camesasca (éd.), Milan, BUR, coll. «Classici», 2007, p. I87.

154. Voir à ce propos Leonid M. Batkin, Gli umanisti italiani. Stile di vita e di pensiero, Rome, Bari, Laterza, I990, p. 44.

155. Giulio Ferroni, Storia della letteratura. Dal Cinquecento al Settecento, Milan, Einaudi Scuola, I991, p. 164 .

156. "[...] far sapere agli altri che l'uomo ha la linea sua da persone virtuose e antichissime», B. Cellini, Vita, ouvr. cité, p. 82. 
aïeux, mais autour de sa propre personne, expliquant que sa vertu artistique lui a permis d'anoblir sa famille ${ }^{157}$. Cellini n'exhorte donc pas les jeunes générations à marcher sur les traces de leurs aînés, mais présente un protagoniste, Benvenuto, qui dépasse ses ancêtres. Et lorsqu'il se donne les traits d'un héros épique (il s'attribue le mérite d'avoir défendu à lui seul le château Saint-Ange, symbole de la chrétienté puisque le pape s’y était réfugié au moment du sac de Rome) ou comme un être tour à tour diabolique et sanctifié, mais toujours surhumain et extra-humain, c'est moins pour proposer un modèle à ses lecteurs que pour se placer dans une perspective d'auto-célébration. Aucune hyperbole, aucun superlatif n'est en effet négligé pour donner au protagoniste de la Vita une aura d'exceptionnalité. Comme si l'individu pouvait désormais être tellement unique qu'il ne pouvait plus être imité, pas plus, d'ailleurs, qu'il ne se place luimême dans une perspective d'imitation, Cellini ne se reconnaissant tributaire que d'un seul artiste, Michel-Ange. 